\title{
Formation of massive black holes in rapidly growing pre-galactic gas clouds
}

\author{
John H. Wise ${ }^{1}$, John A. Regan ${ }^{2}$, Brian W. O’Shea ${ }^{3,4}$, Michael L. Norman $^{5,6}$, Turlough P. Downes ${ }^{2}$ \& Hao Xu ${ }^{5,6,7}$
}

\begin{abstract}
${ }^{1}$ Center for Relativistic Astrophysics, School of Physics, Georgia Institute of Technology, Atlanta, GA 30332, USA; jwise@gatech.edu ${ }^{2}$ Centre for Astrophysics and Relativity, School of Mathematical Sciences, Dublin City University, Dublin, Ireland ${ }^{3}$ Department of Computational Mathematics, Science and Engineering, Michigan State University, East Lansing, MI 48824, USA ${ }^{4}$ Department of Physics and Astronomy, Michigan State University, East Lansing, MI 48824, USA ${ }^{5}$ Center for Astrophysics and Space Sciences, University of California, San Diego, La Jolla, CA 92093, USA ${ }^{6}$ San Diego Supercomputer Center, San Diego, La Jolla, CA 92093, USA ${ }^{7}$ IBM, 2455 South Road,
\end{abstract} Poughkeepsie, NY 12601

The origin of supermassive black holes (SMBHs) that inhabit the centers of massive galaxies is largely unconstrained ${ }^{1,2}$. Remnants from supermassive stars (SMSs) with masses around 10,000 solar masses provide the ideal seed candidates, known as direct collapse black holes ${ }^{3-6}$. However, their very existence and formation environment in the early Universe are still under debate, with their supposed rarity further exacerbating the problem of modeling their ab-initio formation ${ }^{7,8}$. SMS models have shown that rapid collapse, with an infall rate above a critical value, in metal-free haloes is a requirement for the formation of a proto-stellar core which will then form an $\mathrm{SMS}^{9,10}$. Using a radiation hydrodynamics simulation of early galaxy formation ${ }^{11,12}$, we show the natural emergence of metal-free haloes both massive enough, and with sufficiently high infall rates, to form an SMS. We find that haloes that are exposed to both a Lyman-Werner intensity of $J_{\mathrm{LW}} \sim 3 J_{21}{ }^{*}$ and that undergo at least one period of rapid growth early in their evolution are ideal cradles for SMS formation. This rapid growth induces substantial dynamical heating ${ }^{13,14}$, amplifying the existing LymanWerner suppression originating from a group of young galaxies 20 kiloparsecs away. Our results strongly indicate that structure formation dynamics, rather than a critical Lyman-Werner (LW) flux, may be the main driver of massive black hole formation in the early Universe. We find that massive black hole seeds may be much more common in overdense regions of the early Universe than previously considered with a comoving number density up to $10^{-3} \mathrm{Mpc}^{-3}$.

Standard cold dark matter cosmologies predict large-scale structure forms hierarchically. Smaller objects forming at early times subsequently merge and grow into larger objects. The existence of SMBHs ${ }^{15,16}$ with masses around $10^{9} \mathrm{M}_{\odot}\left(\mathrm{M}_{\odot}\right.$, solar mass $)$ only $800 \mathrm{Myr}$ after the Big Bang indicate that there must have been an early intense convergence of mass in rare locations.

We performed a suite of cosmological radiation hydrodynamics simulations (named Renaissance; see Methods) to elucidate the formation of the first generations of stars and galaxies in the Universe $\mathrm{e}^{11,12}$ with the code $\mathrm{ENZO}^{17}$. It includes models for the formation of massive metal-free (Population III; Pop III) stars and subsequent metal-enriched stars not unlike ones found in the Galaxy. We follow the impact of their ionizing radiation ${ }^{18}$ and supernova explosions on their environments as $J_{21}$ is the intensity of background radiation in units of
$10^{-21} \mathrm{erg} \mathrm{cm}^{-2} \mathrm{~s}^{-1} \mathrm{~Hz}^{-1} \mathrm{sr}^{-1}$. galaxies first assemble, both of which play an important role in regulating early galaxy formation.

Motivated by possible early SMS formation, we analyze the region from the Renaissance Simulation suite that is centered on the densest cosmological volume of 133.6 comoving $\mathrm{Mpc}^{3}$ and contains 822 galaxies at its ending redshift of $z=15$ (270 Myr after the Big Bang). We identify candidate SMS host haloes by searching the simulation for metal-free atomic cooling haloes without prior star formation at $z=15$. We place no constraints on the level of LW flux impacting the haloes. There are 670 atomic cooling haloes, ten of which are metalfree and have not hosted prior star formation (see Extended Data Table 1). The remaining atomic cooling haloes have formed stars prompted by either $\mathrm{H}_{2}$ or metal line cooling and are not conducive for SMS (and subsequent direct collapse black hole) formation. Out of these ten haloes, we concentrate on two "target" haloes-the most massive halo $(\mathrm{MMH})$ and, separately, the most irradiated halo ( $\mathrm{LWH})$ - in this study. We resample their mass distributions at $z=20$ at a mass resolution higher by a factor of 169 and resimulate them to study their gravitational collapse in more detail.

Both haloes assemble in a region $10-25 \mathrm{kpc}$ away from a group of young galaxies that have photo-ionized, photo-heated, and chemically enriched their adjacent environments (Fig. 1). At $z=18$, the young galaxies near the MMH (Fig. 1a through 1d) have created an amorphous $\mathrm{H}$ II region with a maximum extent of $20 \mathrm{kpc}$. As the star formation rates grow in these young (massive) galaxies, the LW intensities increase from $J_{\mathrm{LW}} \simeq 1 J_{21}$ at $z=18$ within 5 physical kpc of the galaxies to $30 J_{21}$ at $z=15$. The only other source of LW radiation comes from four nearby haloes hosting Pop III stars, 3$5 \mathrm{kpc}$ from the LWH. Both target haloes are impacted by a LW intensity $J_{\mathrm{LW}} \simeq 3 J_{21}$ at $z=15$ (Fig. 1d, 1h). This total flux impinging on the target haloes is 6-600 times lower than previous critical estimates for SMS formation ${ }^{19-21}$.

The metal-enriched regions around these galaxy congregations only reach a distance of $5 \mathrm{kpc}$, far from the target haloes. These heavy elements originate from both their Pop III progenitors and ongoing star formation in the galaxies. Over the next $60 \mathrm{Myr}$, the ionizing radiation from the young growing galaxies near both target haloes extends the $\mathrm{H}$ II regions to nearly $40 \mathrm{kpc}$ in radius, evident in Fig. 1. This leaves the vast majority of the intergalactic medium and associated collapsed haloes chemically pristine but nonetheless bathed in LW radiation, helping to prevent Pop III star formation.

During the halo assembly process (Fig. 2a), the LW intensity increases from $0.3 J_{21}$ to $3 J_{21}$ at $z=15$ (Fig. 2b), corresponding to a minimum halo mass $M_{\text {crit }} \simeq 3 \times 10^{6} \mathrm{M}_{\odot}$ that can support $\mathrm{H}_{2}$ cooling $^{22}$ and primordial star formation. However, the MMH (LWH) gravitationally collapse only after they reach the atomic cooling limit at $z=16.4(15.3)$, having masses of $2.6(5.8) \times 10^{7} \mathrm{M}_{\odot}$, an order of magnitude above $M_{\text {crit }}$. Upon closer inspection, we find that both target haloes experience a period of intense growth in mass (Fig 2a). The 


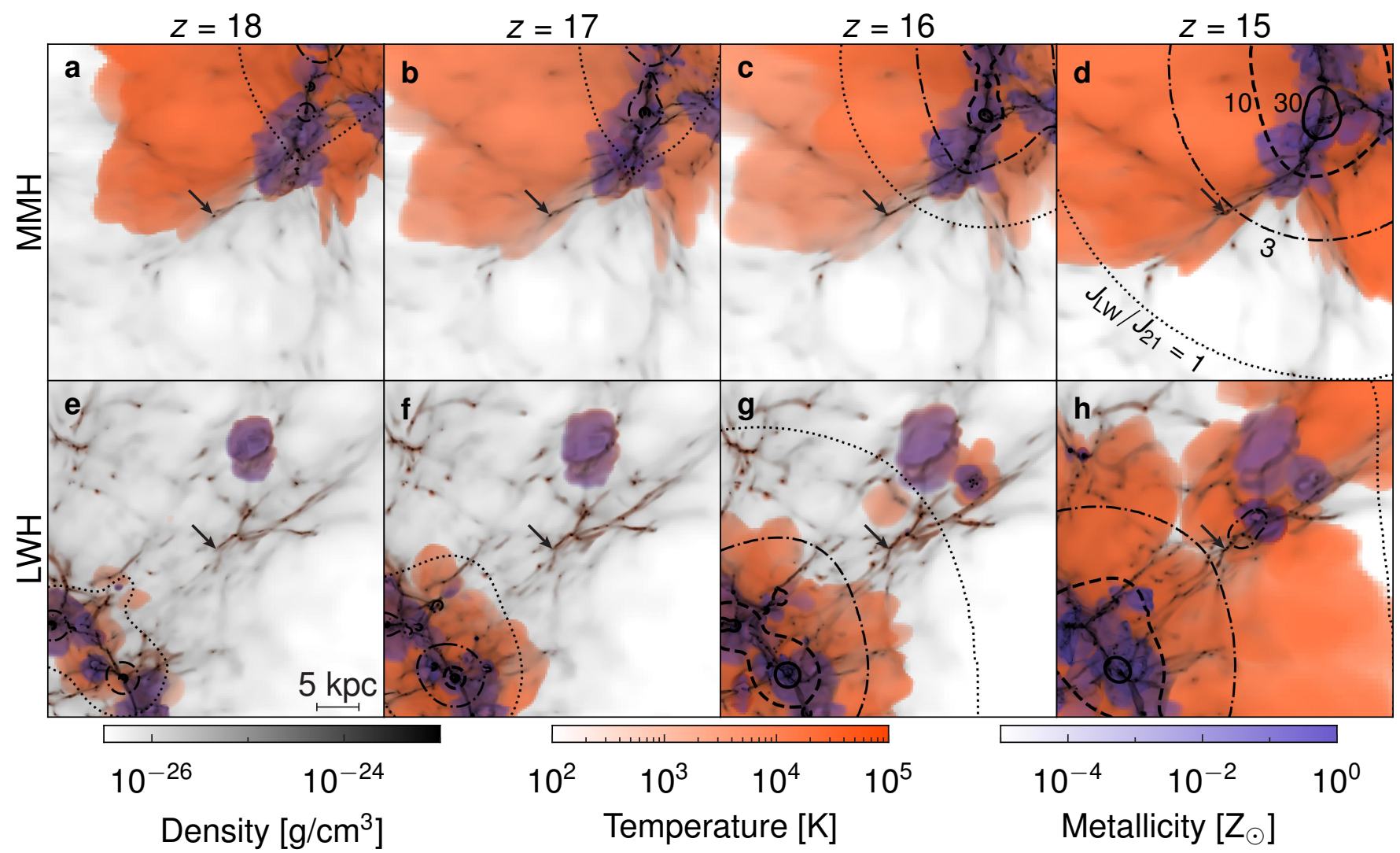

Figure 1 Thermal and chemical evolution of the immediate pre-galactic environment. Projections of temperature (orange), metallicity (blue), and gas density (black) of a region (40 kpc across with a $8 \mathrm{kpc}$ depth) centered on the MMH (a-d) and LWH (e-h) that are highlighted with arrows. Going from left to right shows the heated and metal-enriched volumes around early galaxies and Pop III stars growing from redshift 18 to 15 (62 million years). The dotted, dash-dotted, dashed, and solid contours indicate where the average Lyman-Werner flux is $1,3,10$, and $30 J_{21}$. Both candidate haloes that host massive black hole formation have $J_{21} \simeq 3$, are just outside of the cosmological $\mathrm{H}$ II region, and are still unaffected by any external metal-rich winds.

MMH grows by a factor of 30 over $30 \operatorname{Myr}(z=21-19)$ as it virializes. The LWH experiences two rapid growth events. It first increases from $2 \times 10^{5} \mathrm{M}_{\odot}$ to $3 \times 10^{6} \mathrm{M}_{\odot}$ between $z=19-18(15 \mathrm{Myr})$, at which point its mass fluctuates just above $M_{\text {crit }}$. At redshift $z=15.8$, it then dramatically grows by a factor of nine within a span of 10 Myr. Most of the accreted matter originates from the parent filament, a major merger, and several minor halo mergers. The currently standard cold dark matter paradigm has this unique prediction of intense matter convergence below the atomic cooling limit and is not present in cosmologies that suppress power below this scale. Nevertheless it is rare, only occurring in $\sim 3 \times 10^{-4}$ of haloes (see Methods) with similar masses and existing in an overdense large-scale environment.

Gas within these growing haloes is dynamically heated as it strives for virial equilibrium, whose heating rate is linearly proportional to the halo mass growth rate ${ }^{13}$. Dynamical heating is only important when gas cooling is inefficient, particularly in rapidly growing low-mass haloes. In combination with the LW negative feedback, it can further suppress any attempt at collapse. Both target haloes sustain substantial dynamical heating during their rapid growth events, driven primarily through major mergers. We find that they are the dominant mechanism for preventing Pop III star formation in these haloes.

The simulations follow the evolution of the target haloes until a density of $10^{-15} \mathrm{~g} \mathrm{~cm}^{-3}$, at which point it is certain that a collapsed object will form (see Supplementary Videos). Both haloes form a gravitationally unstable core with a mass and radius of 30,000 $(200,000)$ $\mathrm{M}_{\odot}$ and 3 (15) pc for the MMH (LWH), respectively. The MMH grows gradually after its rapid growth event, allowing the system to form a rotationally supported disk that is comparatively cold at $300 \mathrm{~K}$ to the sur- rounding 10,000 $\mathrm{K}$ gas. The medium within the cold disk is turbulent, which causes numerous weak shocks (Fig. 3d). The disk then fragments into three clumps (Fig. 3a, 3b), as thermal and rotational support cannot counteract their gravitational forces (see Methods), all of which proceed to collapse. The morphology of the LWH is completely different from the MMH because of a recent major merger. The collision causes a sheet-like overdensity (Fig. 3e) that cools to $300 \mathrm{~K}$, becoming gravitationally unstable to fragmentation. A single clump fragments from the sheet (Fig. 3f) and undergoes a catastrophic collapse. All clumps in both target haloes have masses around $1000 \mathrm{M}_{\odot}$.

The radial profiles of the gas density (Fig. 4a) generally follow a power-law with a slope of -2 that is expected for an isothermal collapse, which can be translated into a gas mass enclosed (Fig. 4b). Deviations from a power-law originate from the two other clumps in the $\mathrm{MMH}$, seen as spikes around $1 \mathrm{pc}$, and the sheet-like structure in the $\mathrm{LWH}$, seen as an inflection point around $1 \mathrm{pc}$. The gas inside the Jeans mass (marked with squares) becomes shielded from the extragalactic LW background, allowing for the $\mathrm{H}_{2}$ fraction to increase to $10^{-3}$, sufficient to cool the gas down to $300 \mathrm{~K}$ (Fig. 4c). Inside a radial mass coordinate of $1000 \mathrm{M}_{\odot}$, adiabatic compression heats the gaseous core to $600-800 \mathrm{~K}$.

The key indicator for SMS formation is rapid gas inflow onto the gravitationally unstable core, not the overall Jeans mass. It has been shown that accretion rates over $\sim 0.04 \mathrm{M}_{\odot} \mathrm{yr}^{-1}$ onto a nascent, central core will result in SMS formation ${ }^{9,23}$. Their weak hydrogen ionizing luminosities cannot reverse these strong gas flows ${ }^{24,25}$. The respective infall mass fluxes (Fig. 4d) at the Jeans mass are 0.17 and $2.1 \mathrm{M}_{\odot} \mathrm{yr}^{-1}$ for the MMH and LWH at the final time, above the critical value for 


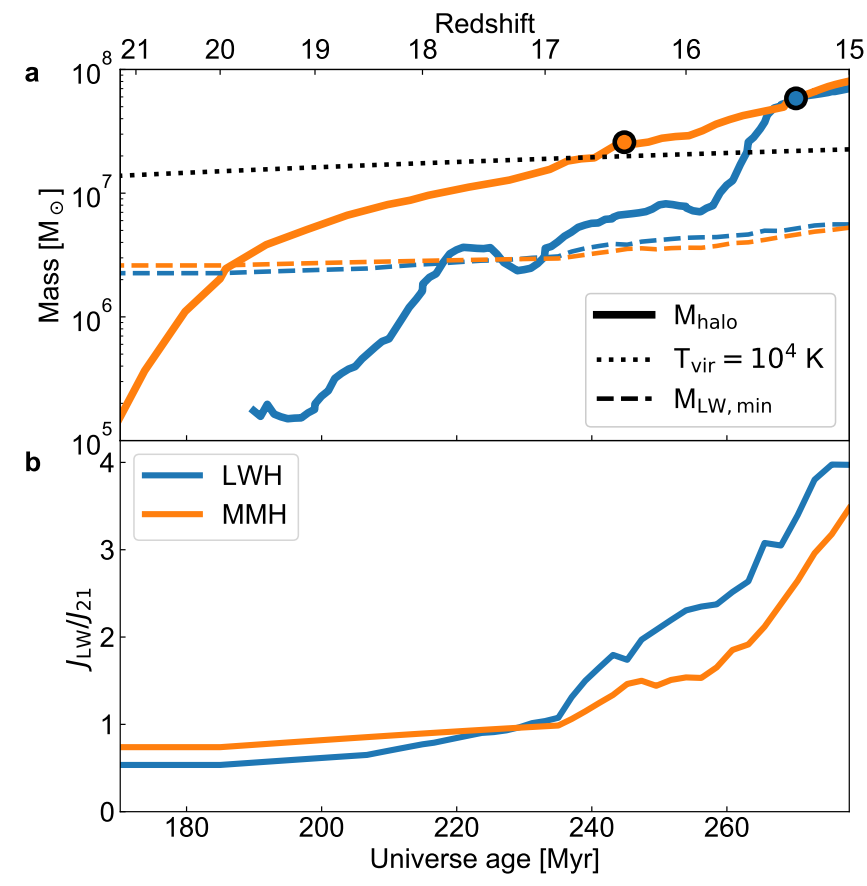

Figure 2 Target halo mass growth histories. The evolution of the metal-free atomic cooling halo with the highest mass (orange) and highest Lyman-Werner flux (blue). a, Halo mass (solid) compared with the minimum mass for $\mathrm{H}_{2}$ cooling (dashed) and atomic cooling (dotted). The circles indicate when the haloes experience gravitational collapse into a massive black hole. The MMH grows smoothly and has a growth history more typical of an atomic cooling halo. In contrast, the LWH undergoes rapid growth just before collapse, growing by a factor of six within 10 million years. b, Lyman-Werner flux at the densest point in units of $J_{21}$ that is increasing from the nearby growing group of young galaxies a distance of $10-15 \mathrm{kpc}$.

SMS formation. This ample supply of inflowing gas provides fuel for the clumps within the central unstable object. The infall rates onto the clumps (Extended Data Fig. 7a) are between 0.03 and $0.08 \mathrm{M}_{\odot} \mathrm{yr}^{-1}$ at their boundaries but increase rapidly to $\sim 0.5 \mathrm{M}_{\odot} \mathrm{yr}^{-1}$ at a radial mass coordinate of $10,000 \mathrm{M}_{\odot}$, suggesting that cores continue to grow rapidly after the final snapshot of our simulation for $1 \mathrm{Myr}$, similar to the typical SMS lifetime (Extended Data Fig. 7b). We thus conclude that the two target haloes will host SMS formation, and subsequently, a direct collapse black hole (DCBH) with an initial seed mass at least $1,000 \mathrm{M}_{\odot}$ and perhaps up to $10,000 \mathrm{M}_{\odot}$ within $1 \mathrm{Myr}$.

Using the formation requirements previously discussed, we can estimate (see Methods) the DCBH formation rate per comoving volume to be $1.1_{-0.91}^{+1.7} \times 10^{-3}$ SMSs per comoving $\mathrm{Mpc}^{-3}$ (68\% confidence interval) that form through this new formation scenario in overdense regions of the Universe. Given that only $0.01-0.1 \%$ of the universe is in such an overdense region, the global number density of DCBH formation is predicted to be $10^{-7}-10^{-6}$ per comoving $\mathrm{Mpc}^{-3}, 100-1000$ times higher than other estimates ${ }^{26}$.

SMSs and thus DCBHs forming in rapidly growing haloes, as proposed here, will be tens of kpc away from the large-scale overdensity. They will take hundreds of Myrs, a substantial fraction of the Universe's age at $z>6$, to fall into the nearby group of galaxies. We predict that these DCBHs will evolve to form the population of faint quasars observed at $z \sim 6$ (ref. 27,28). This population will be within the reach of the James Webb Space Telescope that will provide stringent constraints on their number densities, directly comparable to our results here.
1. Volonteri, M. The Formation and Evolution of Massive Black Holes. Science 337, 544 (2012). 1208.1106.

2. Greif, T. H. The numerical frontier of the high-redshift Universe. Computational Astrophysics and Cosmology 2, 3 (2015). 1410.3482.

3. Omukai, K. Primordial Star Formation under Far-Ultraviolet Radiation. Astrophys. J. 546, 635-651 (2001). astro-ph/0011446.

4. Begelman, M. C., Volonteri, M. \& Rees, M. J. Formation of supermassive black holes by direct collapse in pre-galactic haloes. Mon. Not. R. Astron. Soc. 370, 289-298 (2006). arXiv: astro-ph/0602363.

5. Hosokawa, T., Omukai, K. \& Yorke, H. W. Rapidly Accreting Supergiant Protostars: Embryos of Supermassive Black Holes? Astrophys. J. 778, 178 (2013). 1308.4457.

6. Ardaneh, K. et al. Direct collapse to supermassive black hole seeds with radiation transfer: cosmological haloes. Mon. Not. R. Astron. Soc. 479, 2277-2293 (2018). 1803.03278.

7. Habouzit, M., Volonteri, M., Latif, M., Dubois, Y. \& Peirani, S. On the number density of 'direct collapse' black hole seeds. Mon. Not. R. Astron. Soc. 463, 529-540 (2016). 1601.00557.

8. Chon, S., Hosokawa, T. \& Yoshida, N. Radiation hydrodynamics simulations of the formation of direct-collapse supermassive stellar systems. Mon. Not. R. Astron. Soc. (2018). 1711.05262.

9. Hosokawa, T., Yorke, H. W., Inayoshi, K., Omukai, K. \& Yoshida, N. Formation of Primordial Supermassive Stars by Rapid Mass Accretion. Astrophys. J. 778, 178 (2013). 1308.4457.

10. Umeda, H., Hosokawa, T., Omukai, K. \& Yoshida, N. The Final Fates of Accreting Supermassive Stars. Astrophys. J. Lett. 830, L34 (2016). 1609.04457.

11. O'Shea, B. W., Wise, J. H., Xu, H. \& Norman, M. L. Probing the Ultraviolet Luminosity Function of the Earliest Galaxies with the Renaissance Simulations. Astrophys. J. Lett. 807, L12 (2015). 1503.01110.

12. Xu, H., Wise, J. H., Norman, M. L., Ahn, K. \& O'Shea, B. W. Galaxy Properties and UV Escape Fractions during the Epoch of Reionization: Results from the Renaissance Simulations. Astrophys. J. 833, 84 (2016). 1604.07842 .

13. Yoshida, N., Abel, T., Hernquist, L. \& Sugiyama, N. Simulations of Early Structure Formation: Primordial Gas Clouds. Astrophys. J. 592, 645-663 (2003). arXiv:astro-ph/0301645.

14. Fernandez, R., Bryan, G. L., Haiman, Z. \& Li, M. $\mathrm{H}_{2}$ suppression with shocking inflows: testing a pathway for supermassive black hole formation. Mon. Not. R. Astron. Soc. 439, 3798-3807 (2014). 1401.5803.

15. Mortlock, D. J. et al. A luminous quasar at a redshift of $z=7.085$. Nature 474, 616-619 (2011). 1106.6088 .

16. Bañados, E. et al. An 800-million-solar-mass black hole in a significantly neutral Universe at a redshift of 7.5. Nature 553, 473-476 (2018). 1712. 01860.

17. Bryan, G. L. et al. ENZO: An Adaptive Mesh Refinement Code for Astrophysics. Astrophys. J. Suppl. 211, 19 (2014). 1307.2265.

18. Wise, J. H. \& Abel, T. ENZO+MORAY: radiation hydrodynamics adaptive mesh refinement simulations with adaptive ray tracing. Mon. Not. $R$. Astron. Soc. 414, 3458-3491 (2011). 1012.2865.

19. Shang, C., Bryan, G. L. \& Haiman, Z. Supermassive black hole formation by direct collapse: keeping protogalactic gas $\mathrm{H}_{2}$ free in dark matter haloes with virial temperatures $\mathrm{T}_{\text {vir }}$ ¿ $\operatorname{rsim} 10^{4} \mathrm{~K}$. Mon. Not. R. Astron. Soc. 402, 1249-1262 (2010). 0906.4773.

20. Agarwal, B., Smith, B., Glover, S., Natarajan, P. \& Khochfar, S. New constraints on direct collapse black hole formation in the early Universe. Mon. Not. R. Astron. Soc. 459, 4209-4217 (2016). 1504.04042.

21. Glover, S. C. O. Simulating the formation of massive seed black holes in the early Universe - I. An improved chemical model. Mon. Not. R. Astron. Soc. 451, 2082-2096 (2015). 1501.05960.

22. Machacek, M. E., Bryan, G. L. \& Abel, T. Simulations of Pregalactic Structure Formation with Radiative Feedback. Astrophys. J. 548, 509521 (2001). astro-ph/0007198.

23. Schleicher, D. R. G., Palla, F., Ferrara, A., Galli, D. \& Latif, M. Massive black hole factories: Supermassive and quasi-star formation in primordial halos. Astron. Astrophys. 558, A59 (2013). 1305.5923.

24. Hosokawa, T. et al. Formation of Massive Primordial Stars: Intermittent UV Feedback with Episodic Mass Accretion. Astrophys. J. 824, 119 (2016). 1510.01407.

25. Sakurai, Y., Hosokawa, T., Yoshida, N. \& Yorke, H. W. Formation of primordial supermassive stars by burst accretion. Mon. Not. R. Astron Soc. 452, 755-764 (2015). 1505.03954.

26. Hirano, S., Hosokawa, T., Yoshida, N. \& Kuiper, R. Supersonic gas streams enhance the formation of massive black holes in the early universe. Science 357, 1375-1378 (2017). 1709.09863. 

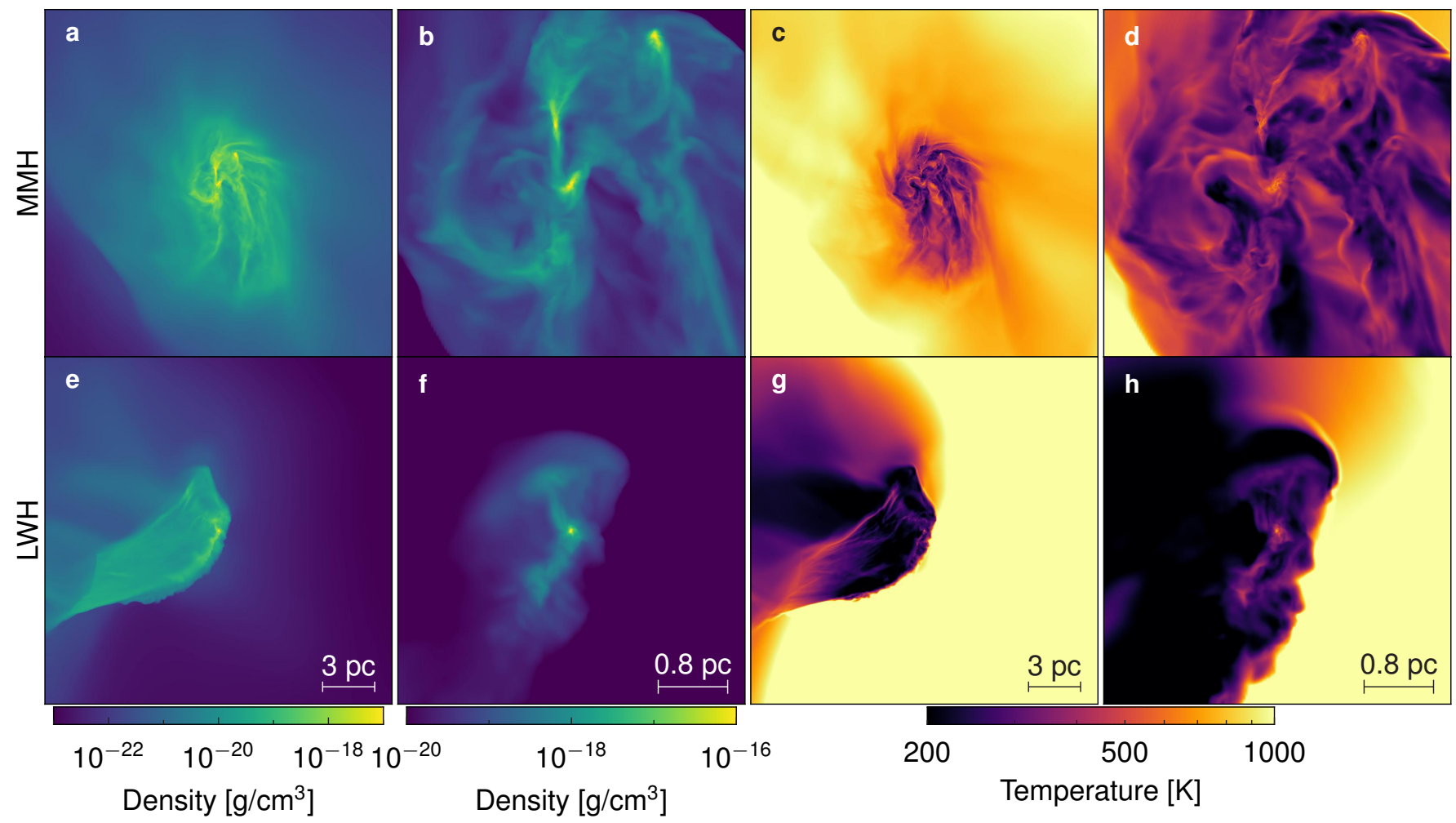

Figure 3 | Morphology of the collapsing objects. Density-weighted projections of gas density (a,b,e,f) and temperature (c,d,g,h) of the MMH (top row) and LWH (bottom row) at the end of the simulations, centered on the densest point and aligned to be perpendicular with the angular momentum vector of the disk. The MMH forms a cold disk that fragments into three clumps, accompanied by weak turbulent shocks within the disk. In the LWH, a dense and cold sheet forms after the collision of two progenitor haloes, where a single clump collapses after becoming self-gravitating. Within a radius of 0.1 parsecs in all of the clumps, adiabatic compression heats the gas.

27. Onoue, M. et al. Minor Contribution of Quasars to lonizing Photon Budget at $z \sim 6$ : Update on Quasar Luminosity Function at the Faint End with Subaru/Suprime-Cam. Astrophys. J. Lett. 847, L15 (2017). 1709.04413.

28. Kim, Y. et al. The Infrared Medium-deep Survey. IV. The Low Eddington Ratio of A Faint Quasar at $\mathrm{z} \sim 6$ : Not Every Supermassive Black Hole is Growing Fast in the Early Universe. Astrophys. J. 855, 138 (2018) 1802.02782.

29. Turk, M. J. et al. yt: A Multi-code Analysis Toolkit for Astrophysical Simulation Data. Astrophys. J. Suppl. 192, 9 (2011). 1011.3514.

Acknowledgements JHW thanks Andrew Benson for assistance with the code GALACTICUS. JHW was supported by NSF awards AST-1614333 and OAC-1835213, NASA grant NNX17AG23G, and Hubble theory grant HSTAR-14326. JR acknowledges support from the EU commission via the Marie Skłodowska-Curie Grant - "SMARTSTARS" - grant number 699941. BWO was supported in part by NSF awards PHY-1430152, AST-1514700, OAC 1835213, by NASA grants NNX12AC98G, NNX15AP39G, and by Hubble theory Grants HST-AR-13261.01-A and HST-AR-14315.001-A. MLN was supported by NSF grants AST-1109243, AST-1615858, and OAC-1835213. The simulation was performed on Blue Waters operated by the National Center for Supercomputing Applications (NCSA) with PRAC allocation support by the NSF (awards ACl-0832662, ACl-1238993, ACl-1514580). The subsequent analysis and the resimulations were performed with NSF's XSEDE allocation AST-120046 on the Stampede2 resource. This research is part of the Blue Waters sustained-petascale computing project, which is supported by the NSF (awards OCl-0725070, ACl-1238993) and the state of Illinois. Blue Waters is a joint effort of the University of Illinois at Urbana-Champaign and its NCSA. The freely available astrophysical analysis code $y t^{29}$ and plotting library matplotlib was used to construct numerous plots within this paper. Computations described in this work were performed using the publicly-available ENZO code, which is the product of a collaborative effort of many independent scientists from numerous institutions around the world.

Author contributions JHW and JR conceived the idea, performed the analysis, and drafted the paper. The Renaissance Simulations were conducted by
$\mathrm{HX}$ and $\mathrm{JHW}$, and the resimulations of the target haloes were conducted by JHW. BWO performed the Monte Carlo analysis for the number density estimate. All authors contributed to the interpretation of the results and to the text of the final manuscript.

Competing Interests The authors declare no competing interests.

Additional information

Extended data is available in the online version of the paper.

Supplementary videos are available in the online version of the paper.

Reprints and permissions information is available at www. nature.com/ reprint.

Correspondence and requests for materials should be addressed to JHW. 

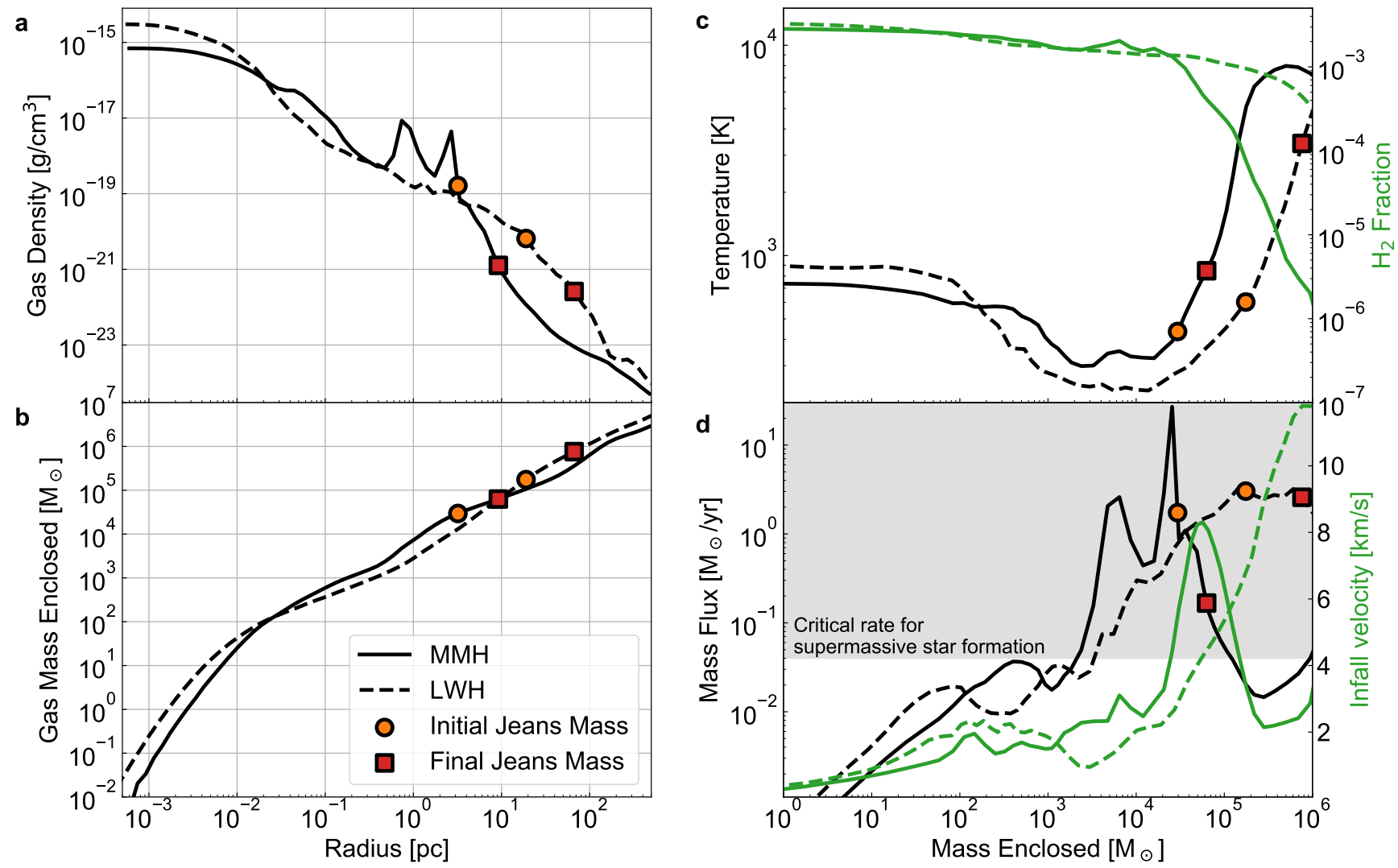

Figure 4 Gravitational collapse of the target haloes. Radially averaged profiles of gas density (a), gas mass enclosed (b), gas temperature (c, black lines), $\mathrm{H}_{2}$ fraction (c, green lines), radial mass infall rates (d, black lines), and radial infall velocities (d, green lines) in the MMH (solid) and LWH (dashed). The orange circles indicate the Jeans mass and associated quantities at that mass scale when the object first becomes gravitationally unstable, whereas the red squares represent the same quantities at the end of the simulation. At the surface of the gravitationally unstable core, gas is infalling at rates upwards of 0.1 solar masses per year, where values above 0.04 solar masses per year can induce and fuel supermassive star formation.

METHODS Cosmological simulation of early galaxy formation. The Renaissance Simulations were carried out using the open source adaptive mesh refinement code $\mathrm{ENZO}^{17}$, a physics rich, highly adaptive code that has been well tuned for high-redshift structure formation simulations ${ }^{11,30-32}$. The Renaissance Simulations have been well-detailed previously in the literature ${ }^{11,12,33-37}$, and here we only summarize the simulation characteristics relevant to this study. All of the Renaissance simulations were carried out in a comoving volume of $(40 \mathrm{Mpc})^{3}$. We set the cosmological parameters using the 7-year WMAP $\Lambda$ CDM+SZ+LENS best fit ${ }^{38}: \Omega_{\mathrm{m}}=0.266, \Omega_{\Lambda}=$ $0.734, \Omega_{\mathrm{b}}=0.0449, h=0.71, \sigma_{8}=0.81$ and $n=0.963$. The simulations were initially run until a redshift $z=6$ at relatively coarse resolution with $512^{3}$ particles each with a mass of $1.7 \times 10^{7} \mathrm{M}_{\odot}$. Three regions of interest were then selected for re-simulation at much higher refinement namely a rare-peak region, a normal region and a void region. The rare-peak region has a comoving volume of $133.6 \mathrm{Mpc}^{3}$, whereas the normal and void regions each have comoving volumes of $220.5 \mathrm{Mpc}^{3}$.

In this study, we focus on the rare-peak simulation, which was selected by extracting the Lagrangian region centered on two $3 \times 10^{10} \mathrm{M}_{\odot}$ haloes at $z=6$, the most massive at that time. The dimensions of the rare-peak region were set at $5.2 \times 7.0 \times 8.3 \mathrm{Mpc}^{3}$. The simulation was re-initialized using MUSIC ${ }^{39}$ with a further three nested grids centered on the rare-peak region. This led to an effective resolution of $4096^{3}$ and a dark matter particle resolution of $2.9 \times 10^{4} \mathrm{M}_{\odot}$ within the highest refinement region. During the course of the simulation further adaptive refinement is allowed within the refinement zone (i.e. the Lagrangian region of the rare-peak) up to a maximum level of 12 leading to a maximum spatial resolution of 19 comoving $\mathrm{pc}(1.2$ proper $\mathrm{pc}$ at $z=15)$. The simulation was halted at a final redshift $z=15$ due to the high computational expense. The halo mass function is well-resolved down to $2 \times 10^{6} \mathrm{M}_{\odot}$ with 70 particles per halo ${ }^{12}$, and at the ending redshift, the simulations contained 822 galaxies having at least 1,000 particles $\left(M_{\text {halo }} \simeq 2.9 \times 10^{7} \mathrm{M}_{\odot}\right)$. We follow the ionization states of hydrogen and helium with a 9-species primordial non-equilibrium chemistry and cooling network ${ }^{40}$, supplemented by metal-dependent cooling tables ${ }^{41}$. Dark matter halo catalogs and the associated merger trees were created with ROCKSTAR $^{42}$ and CONSISTENT-TREES ${ }^{43}$, respectively.

Star formation and feedback. The Renaissance Simulations include both self-consistent Pop III and metal-enriched star formation at the maximum refinement level. The simulation captures star formation in haloes as small as $3 \times 10^{6} \mathrm{M}_{\odot}$ (ref. 33). Pop III star formation occurs when a cell meets all of the following criteria:

- An overdensity of $5 \times 10^{5}\left(\sim 10^{3} \mathrm{~cm}^{-3}\right.$ at $\left.\mathrm{z}=10\right)$

- A converging gas flow $\left(\nabla \cdot \mathrm{v}_{\text {gas }}<0\right)$

- A molecular hydrogen fraction $\mathrm{f}_{\mathrm{H} 2}>5 \times 10^{-4}$

These physical conditions are typical of collapsing metal-free molecular clouds $\sim 10$ Myr before the birth of a Pop III main sequence $\operatorname{star}^{30}$. In this scenario, each star particle within the simulation represents a single star. Population III star formation occurs if the metallicity is less than $10^{-4}$ of the solar fraction in the highest density cell with metal-enriched star formation proceeding otherwise. Pop III star formation furthermore requires that the $\mathrm{H}_{2}$ fraction is greater than $5 \times 10^{-4}$. This takes into account the fact that star formation should not proceed in the presence of a strong Lyman-Werner (LW) radiation field. The functional form of the IMF and supernovae feedback are calibrated ${ }^{44}$ against high-resolution Pop III star formation simulations, stellar evolution models, and observations and models of star formation in local molecular clouds. Stellar feedback uses the MORAY radiative 
transport framework ${ }^{18}$ for ionizing photons. LW radiation that dissociates $\mathrm{H}_{2}$ is modeled using an optically thin, inverse square law profile, centered on all star particles. We do not include any $\mathrm{H}_{2}$ self-shielding, which only becomes important at high densities. In particular, DCBH host halo candidates only shield themselves from a background at scales below $3 \mathrm{pc}^{45}$, which is close to our resolution limit. A LW background radiation field is also included to model radiation from stars which are not within the simulation volume ${ }^{44}$ that is added to the spatially varying LW radiation field created by stars inside the volume. In the high density region of the rare-peak simulation the LW radiation from stars dominates over the background. Although we cannot follow Pop III star formation in haloes below $3 \times 10^{6} \mathrm{M}_{\odot}$, it is suppressed by the LW background in such haloes ${ }^{22,46,47}$ and also by baryonic streaming velocities in certain regions ${ }^{26,48}$. Thus, we are confident that our simulation follows the complete star formation history of all collapsed structure, and thus the metal-enrichment history of pre-galactic gas that is vital to determine the conditions for DCBH formation.

Direct-collapse black holes. The star formation and feedback models do not include a DCBH formation model but consider the appropriate astrophysical processes to ascertain the chemical and thermal state of all collapsed objects resolved by the simulation, essential for searching for candidate DCBH formation sites. Also critical to any DCBH formation scenario is the inclusion of Population III star formation and its supernova feedback that generally enriches typical pre-galactic material with heavy elements. The emergence of a DCBH/SMS environment is therefore a robust prediction of the Renaissance simulations. The dark matter resolution in the original Renaissance Simulations is not sufficient to follow the detailed collapse of these objects ${ }^{49}$, however the resimulations (see next section) of the target haloes have an ultimate mass and spatial resolution of $103 \mathrm{M}_{\odot}$ and $60 \mathrm{AU}$, allowing us to accurately follow the dynamics of the collapsing halo and determine whether their gas infall rates are large enough to support SMS and thus DCBH formation.

High-resolution simulations of target haloes. After identifying the candidate haloes in the Renaissance Simulations, we resimulate the two target haloes at higher mass and spatial resolution, starting at a redshift $z=20$. We first identify the dark matter particles within three virial radii of the target haloes at the final redshift $z=15$. Using their unique particle identifiers, we determine their positions at $z=20$ and split their mass equally into 13 particles. Twelve of child particles are placed at the 12 vertices of a hexagonal close packed array, and the remaining particle is placed at the original particle position ${ }^{50}$. We recursively split the particles twice, decreasing the dark matter particle mass by a factor of $13^{2}$ to $103 \mathrm{M}_{\odot}$ in the Lagrangian region of the target haloes. This method of particle splitting is widely used in high-resolution cosmological simulations ${ }^{49,51-53}$, however this method may induce artificial smoothing of the density field ${ }^{54}$ and does not add additional small-scale power to the matter distribution.

The Renaissance Simulations show that the target haloes remain metalfree and are not exposed to any ionizing radiation. Their thermodynamic evolution and collapse are thus primarily controlled by the halo growth history and the impinging LW flux. With these priors, we can safely neglect star formation and feedback, focusing on the dynamics of their collapse. We impose a uniform LW radiation background that is time-dependent and is taken from the flux measured at the center of the most massive progenitor halo in the original simulation (see Fig. 2b). Spatial deviations from this value are less than a few percent within the Lagrangian region and does not affect the thermodynamics of the gas.

In addition to the increased mass resolution, we increase the spatial resolution by resolving the local Jeans length by at least 16 cells, whereas the original simulation did not have enough mass resolution to enforce such a refinement criterion. We use this initial resimulation to identify the time of collapse. We then restart the simulations a free-fall time (approximately $20 \mathrm{Myr}$ ) before the collapse time, further increasing the spatial resolution so that the Jeans length is resolved by at least 64 cells. Computational limitations restrict us from running at such high resolution for the entirety of the resimulation, thus we only increase the resolution during the final stages of the collapse. We also include $\mathrm{H}_{2}$ self-shielding of the LW radiation field during this final resimulation ${ }^{55}$ and compute the primordial cooling rates with the software library GRACKLE ${ }^{56}$. We enforce a maximum refinement level of 24 , corresponding to a comoving (physical at $z=15$ ) resolution

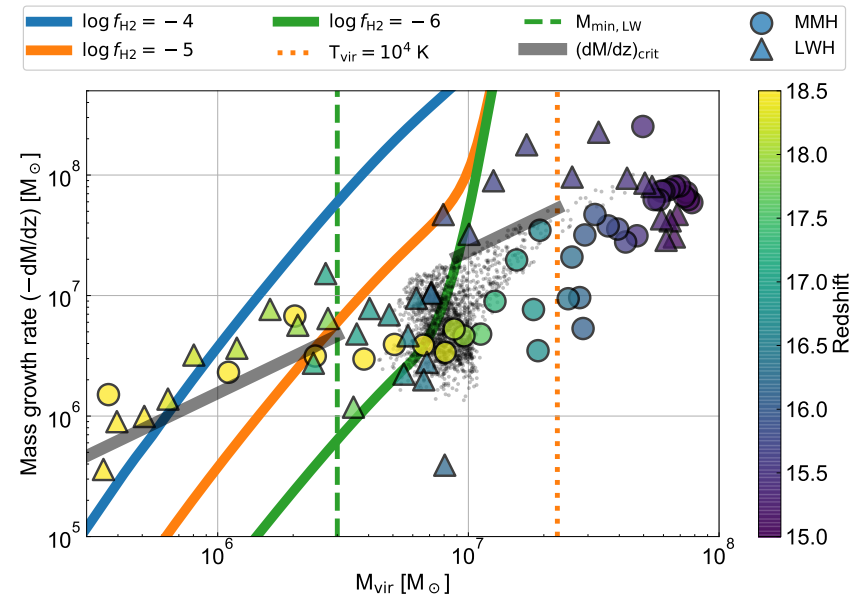

Extended Data Figure 1 | Simulated and critical halo mass growth rates for SMS formation. A halo conducive for SMS formation must grow to the atomic cooling limit $\left(2.2 \times 10^{7} \mathrm{M}_{\odot}\right.$ at redshift 15 ; orange dotted line $)$ without hosting star formation or being chemically enriched from nearby galaxies. Haloes with masses below minimum mass $M_{\mathrm{min}, \mathrm{LW}}$ (dashed green line) are suppressed by an external Lyman-Werner radiation field. Above this mass, haloes with sufficient dynamical heating to suppress radiative cooling grow above a critical rate (Eq. 2), which are shown for $\mathrm{H}_{2}$ number fractions of $10^{-4}$ (blue solid line), $10^{-5}$ (orange solid line), and $10^{-6}$ (green solid line). The simulated growth rates of the MMH (circles) and LWH (triangles) are above the $10^{-6}$ rate once it passes $M_{\min , \mathrm{LW}}$. Above a halo mass of $8 \times 10^{6} \mathrm{M} \odot$ (a virial temperature of $8000 \mathrm{~K}$ at redshift 15), dynamical heating will not suppress cooling as the atomic radiative cooling rates are several orders of magnitude higher than molecular ones. Both halos rapidly grow to $M_{\min , \mathrm{LW}}$ that causes dynamical heating, preventing collapse for a sound-crossing time. The LWH grows from $8 \times 10^{6} \mathrm{M}_{\odot}$ to the atomic cooling limit within a dynamical time of the central core. Both conditions set a critical growth rate (thick solid gray lines). All other atomic cooling haloes (gray points) have similar growth rates between halo masses of $M_{\mathrm{min}, \mathrm{LW}}$ and $8 \times 10^{6} \mathrm{M}_{\odot}$ but far short of this critical growth rate. Nearly all of these haloes cool and form stars before reaching the atomic cooling threshold.

of $960 \mathrm{AU}(60 \mathrm{AU})$. We stop the resimulations once they reach this maximum refinement level. We smooth the dark matter density field at scales below 9.5 comoving pc ( 0.6 physical pc at $z=15$; refinement level 13). At these scales, the gas density dominates the matter density, and by smoothing the dark matter density, we remove any artifacts associated with the discrete representation of the dark matter mass distribution ${ }^{30}$.

Dynamical heating. For a halo to potentially host a SMS/DCBH, it must grow to the atomic cooling limit without forming stars or being chemically enriched, thus any efficient cooling must be suppressed. LW radiation can suppress $\mathrm{H}_{2}$ formation, and dynamical heating can counteract any $\mathrm{H}_{2}$ cooling in low-mass haloes. The dynamical heating rate $^{13}$ is given by

$$
\Gamma_{\mathrm{dyn}}=\alpha M_{\text {halo }}^{-1 / 3} \frac{k_{\mathrm{B}}}{\gamma-1} \frac{d M_{\text {halo }}}{d t},
$$

where $\alpha$ is a coefficient ${ }^{57}$ relating the halo virial mass and temperature ( $\left.T_{\text {halo }}=\alpha M_{\text {halo }}^{2 / 3}\right), M_{\text {halo }}$ is the total halo mass, $k_{\mathrm{B}}$ is the Boltzmann constant, and $\gamma=5 / 3$ is the adiabatic index. This process is only relevant when radiative cooling rates $\Lambda$ ( $\left.T_{\text {halo }}\right)$ per hydrogen atom have similar values. Below the atomic cooling limit, chemically primordial haloes rely on the inefficient coolant $\mathrm{H}_{2}$ to collapse and form stars. Radiative cooling will thus be suppressed when $\Gamma_{\mathrm{dyn}}>n \Lambda$, where $n$ is the hydrogen number density. Given this inequality, this sets a critical halo growth rate ${ }^{13}$

$$
\left(\frac{d M_{\text {halo }}}{d t}\right)_{\text {crit }}=\frac{2 \alpha}{3} \frac{\gamma-1}{k_{\mathrm{B}}} M_{\text {halo }}^{-1 / 3} n \Lambda\left(T_{\text {halo }}\right)
$$

above which cooling is suppressed. Extended Data Fig. 1 compares the critical growth rates (colored lines) for three different $\mathrm{H}_{2}$ fractions, using Eq. 2, and the growth rates of the two target haloes (circles and triangles). 


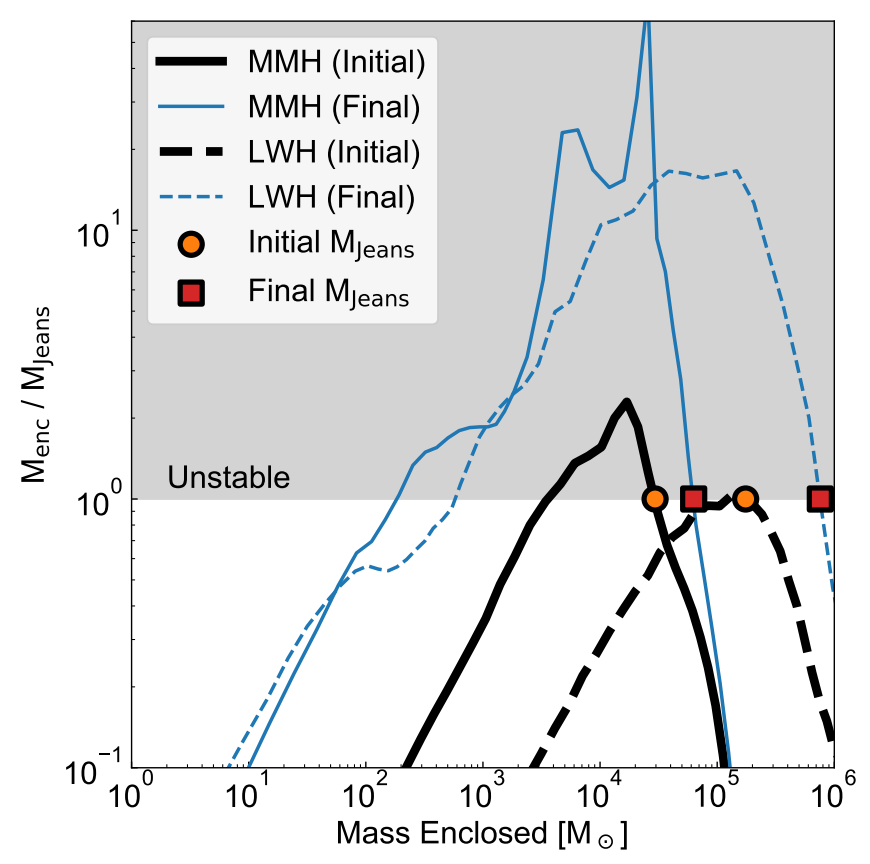

Extended Data Figure 2 Gravitational instability of the growing core. The ratio of the enclosed gas mass $M_{\mathrm{enc}}$ and the Jeans mass $M_{\mathrm{J}}$ as a function enclosed gas mass is shown when the halo first becomes gravitationally unstable (thick black lines) when $M_{\mathrm{enc}} / M_{\mathrm{J}} \geq 1$ (shaded region) and the final simulation state (thin blue lines) for the MMH (solid lines) and LWH (dashed lines). The orange circles and red squares indicate the mass scale of the collapsing gas cloud that is co-located with the center of the host halo.

We calculate the primordial cooling rate $\Lambda(T)$ for each $\mathrm{H}_{2}$ fraction with GRACKLE $^{56}$. Typical $\mathrm{H}_{2}$ number fractions $f_{\mathrm{H} 2}$ in the target halo centers prior to collapse are between $10^{-5}$ and $10^{-6}$.

Absent of any dynamical heating, haloes can cool and collapse through $\mathrm{H}_{2}$ once they reach a critical mass ${ }^{22,47}$

$$
\frac{M_{\mathrm{min}, \mathrm{LW}}}{\mathrm{M}_{\odot}}=1.25 \times 10^{5}+2.65 \times 10^{5}\left(\frac{J_{\mathrm{LW}}}{J_{21}}\right)^{0.47}
$$

shown as the dashed vertical line for $J_{\mathrm{LW}}=1 J_{21}$, the intensity at both target haloes when they have such a mass. Atomic hydrogen cooling becomes dominant over molecular cooling at $T \simeq 7000 \mathrm{~K}$ (corresponding to a halo mass of $6 \times 10^{6} \mathrm{M}_{\odot}$ at $z=15$ ), indicated by the sharp rise in the critical curves, after which dynamical heating becomes unimportant in the target haloes. In any chemically pristine halo unaffected by ionizing radiation, gravitational collapse ensues above this mass within a freefall timescale. Models of SMS formation require infall rates above $\sim 0.04$ $\mathrm{M}_{\odot} \mathrm{yr}^{-1}$ that are only driven by deep gravitational potentials of atomic cooling haloes, whose mass is shown as the dotted vertical line at a mass of $2.2 \times 10^{7} \mathrm{M}_{\odot}[(1+z) / 16]^{-3 / 2}$.

Both the MMH and LWH rapidly assemble as it virializes, initially growing at a rate $(-\mathrm{dM} / \mathrm{dz})$ of $(1-3) \times 10^{6} \mathrm{M}_{\odot}$ (per unit redshift) when it has a mass $M \leq 10^{6} \mathrm{M} \odot$. Although $\mathrm{H}_{2}$ formation is photo-suppressed at these low masses, dynamical heating is present in these early rapid periods of growth and have lasting effects on the halo gas. The infalling gas shocks near the halo center, heating the halo to the virial temperature. Only after a sound-crossing time, the halo comes into virial equilibrium. This takes $30 \mathrm{Myr}$ for a $3 \times 10^{6} \mathrm{M}_{\odot}$ halo (150 pc radius) and a sound speed of 10 $\mathrm{km} / \mathrm{s}$.

As the haloes equilibrate, they continue to grow but at a reduced rate, however still above the critical $f_{\mathrm{H} 2}=10^{-6}$ curve until a mass of $6 \times$ $10^{6} \mathrm{M}_{\odot}$. The MMH accretes at a near constant rate of $3 \times 10^{6} \mathrm{M}_{\odot}$ (per unit redshift) with a peak rate of $7 \times 10^{6} \mathrm{M}_{\odot}$ (per unit redshift) at $M=$ $2 \times 10^{6} \mathrm{M}_{\odot}$. The LWH growth rate fluctuates between $10^{6}$ and $10^{7} \mathrm{M}_{\odot}$ (per unit redshift). When atomic cooling becomes efficient, catastrophic collapse occurs within a free-fall time $t_{\mathrm{ff}}=\sqrt{3 \pi / 32 G \rho}$ that is $20 \mathrm{Myr}$ for a density of $10^{-23} \mathrm{~g} \mathrm{~cm}^{-3}$, typical of haloes compressed adiabatically ${ }^{58}$.

As shown in Fig. 2, the MMH undergoes one rapid growth event, starting at $2 \times 10^{5} \mathrm{M}_{\odot}$ at $z=21$ as it viralizes and occuring before $\mathrm{H}_{2}$ cooling becomes efficient (and hence before the local LW flux becomes important). The rapid infall creates a shock near its center, heating the halo gas over a sound-crossing time of $30 \mathrm{Myr}$. The halo gas stabilizes after this event, and then it collapses on a free-fall time $t_{\mathrm{ff}}=\sqrt{3 \pi / 32 G \rho} \simeq 20 \mathrm{Myr}$, where $\rho \simeq 10^{-23} \mathrm{~g} \mathrm{~cm}^{-3}$ is the maximum gas density before collapse. After the rapid growth halts, the halo must grow to the atomic cooling limit within $50 \mathrm{Myr}$, the sum of the sound-crossing time and free-fall time. Otherwise, it will collapse when atomic cooling is not efficient, and it will cool through $\mathrm{H}_{2}$ and fragment into more typical Population III star formation. It collapses at $z=16.4$ when its mass is just above the atomic cooling limit. There exists $75 \mathrm{Myr}$ between these two redshifts, leaving $25 \mathrm{Myr}$ for early rapid growth. Given that halo masses increase exponentially ${ }^{59}$, i.e. $d M / d z \propto \alpha M$ with $M(z) \propto e^{\alpha z}$, we can estimate a critical growth rate in those first $25 \mathrm{Myr}$, shown as a left thick gray line in Extended Data Fig. 1, using the mass difference between $z=21-19.2(25 \mathrm{Myr})$ when it grows to $3 \times 10^{6} \mathrm{M}_{\odot}$. The LWH experiences two rapid growth episodes, where the first one is similar to the MMH. Afterwards, its mass hovers just above the critical value $M_{\text {crit }}$ for efficient $\mathrm{H}_{2}$ cooling. The later growth spurt occurs just before it starts to cool atomically, seen by the increase in $(-\mathrm{dM} / \mathrm{dz})$ to greater than $3 \times 10^{7} \mathrm{M}_{\odot}$. The associated dynamical heating delays the collapse of the LWH until its mass is $5.8 \times 10^{7} \mathrm{M}_{\odot}$, twice the atomic cooling limit.

In general for dynamical heating to suppress metal-free star formation, halo growth must be rapid from the $\mathrm{H}_{2}$ cooling limit to the atomic cooling limit. More specifically, it must happen faster than a free-fall time (20 Myr). This sets another, more general, critical growth rate between these two halo mass regions, using the same approach as before. It is shown as the thick gray line between the green $f_{\mathrm{H} 2}=10^{-6}$ critical curve and the atomic cooling limit. Any halo that grows faster than this rate will have its gravitational potential deepen faster than it can collapse, making it more likely to support strong radial inflows, conducive for SMS formation.

Gravitational collapse of the central core. After reaching the atomic cooling threshold, the gas begins to cool rapidly and (dynamical) heating mechanisms are suppressed. Above a temperature of approximately $8000 \mathrm{~K}$, radiative cooling of neutral hydrogen sets an upper limit to the temperature of the halo inside the virial radius, inducing a rapid increase in density and ultimately gravitational collapse. Extended Data Fig. 2 shows the ratio of the enclosed mass, $M_{\mathrm{enc}}$, and the Jeans mass, $M_{\mathrm{J}}$, against the enclosed gas mass. For gravitational collapse to take hold, the gas inside the collapsing core must exceed the Jeans mass of the gas. In Extended Data Fig. 2, any lines entering the shaded region are unstable to gravitational collapse. For the MMH, the core of the halo first becomes unstable with an enclosed mass approximately $3 \times 10^{4} \mathrm{M}_{\odot}-$ marked by an orange circle. As the halo grows in mass, the region grows to almost $10^{5} \mathrm{M}_{\odot}$ by the end of the simulation, which is gravitationally unstable and in principal also subject to fragmentation. The LWH first crosses into a region of instability with an enclosed mass of greater than $10^{5} \mathrm{M}_{\odot}$, and by the end of the simulation, the central region of almost $10^{6} \mathrm{M}_{\odot}$ has become unstable to gravitational collapse.

Support within the collapsing core. As the core regions of both haloes start to undergo gravitational collapse, as outlined above, both thermal, turbulent, and rotational support will act to counteract the gravitational collapse. If there is sufficient gas support, the gravitational collapse can be suppressed even though the Jeans mass has been breached. Extended Data Fig. 3 shows both the thermal and turbulent sound speeds as a function of enclosed mass. The thermal sound speed is calculated in the usual way as

$$
c_{\mathrm{s}}=\sqrt{\frac{\gamma k_{\mathrm{B}} T}{\mu m_{\mathrm{H}}}}
$$

where $c_{\mathrm{S}}$ is the sound speed, $\gamma$ is the adiabatic index, $k_{\mathrm{B}}$ is the Boltzmann constant, $T$ is the temperature, $\mu$ is the mean molecular weight and $m_{\mathrm{H}}$ is the hydrogen mass. In calculating the total pressure support of the gas, the impact of the turbulent velocity field must also be considered. We calculate the root mean squared turbulent velocities of the gas by subtracting the bulk 


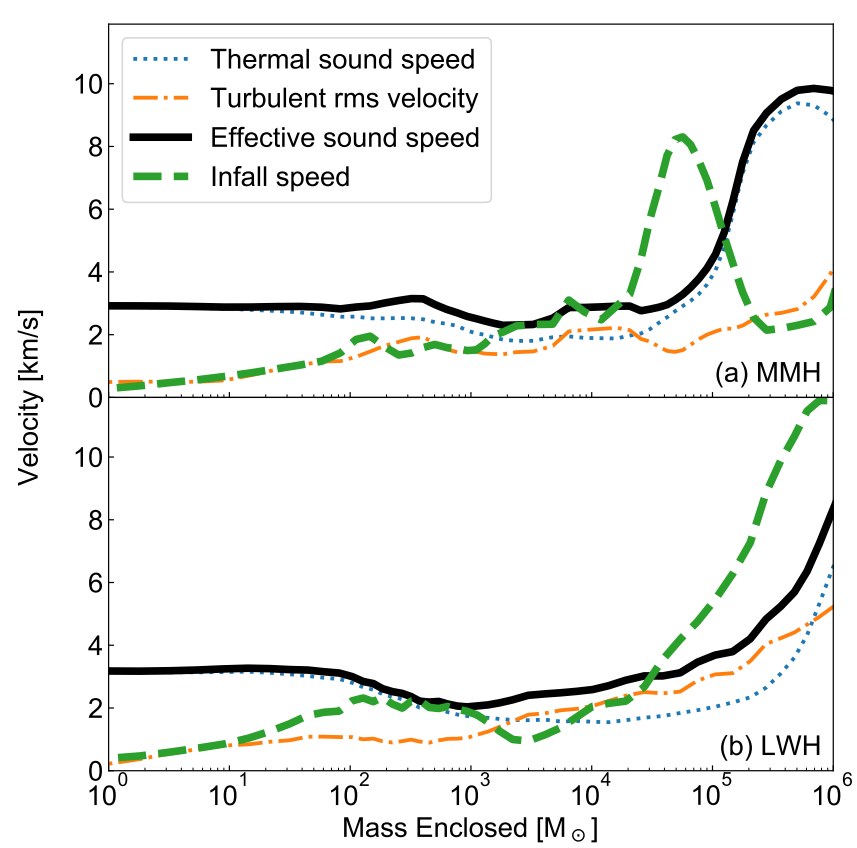

Extended Data Figure 3 | Thermal and turbulent support of the collapsing core. Gravitational forces dominate over thermal and turbulent internal pressures within the collapsing core in the MMH (a) and the LWH (b). The thermal sound speed (blue dotted lines) and turbulent rms velocity (orange dash-dotted line) both contribute to the effective sound speed (black solid line) that provides partial resistance to a catastrophic collapse. The radial infall speed (green dashed line) shows that the flow becomes supersonic at the Jeans mass scale and then transitions to a subsonic flow at smaller mass scales. In the LWH, the radial inflow becomes transonic at a mass scale of 1000 solar masses.

gas velocity from the velocity field. We compute the bulk velocity as the velocity of the native computational cells averaged over a spherical $32^{3}$ grid that has cell widths much coarser than the simulation data. It has an outer radius equal to the virial radius, equally spaced angular bins, and equally logspaced radial bins. We then approximate the bulk velocity of each native cell with the value of the spherical grid cell that contains the native cell center.

The turbulent and thermal components act together to support the gas against collapse and create an effective sound speed, $c_{\mathrm{eff}}^{2}=c_{\mathrm{s}}^{2}+v_{\mathrm{rms}}^{2}$ In Extended Data Fig. 3, we see that the infall speed always exceeds the effective sound speed at some enclosed mass, indicating that, at this scale, the thermal and turbulent support cannot support the gas against gravitational collapse. For the MMH, the infall speed exceeds the effective sound speed at approximately $10^{5} \mathrm{M}_{\odot}$. This behavior is similar to the scale at which the gas also becomes Jeans unstable, and so we define the collapsing core radius to be equal to this scale. For the LWH, the gas provides little or no thermal or turbulent support, and the gas is free to collapse on approximately the freefall timescale.

Interestingly in both cases, the radial inflow becomes transonic at scales between $10^{3} \mathrm{M}_{\odot}$ and $10^{4} \mathrm{M}_{\odot}$. This indicates that at mass scales greater than approximately $10^{3} \mathrm{M}_{\odot}$ any fragments are thermally and/or turbulently supported, and we do not expect fragmentation of the gas cloud below this scale. Any protostars forming within these clouds would have most of the gas contained in the fragment available for accretion. We will examine more closely the possible fragmentation of the outer core into gas clouds in the next subsection.

Extended Data Fig. 4 shows the rotational support provided by the gas against gravitational collapse. The rotational velocity of the gas is calculated by $|j| / a_{1}$, the ratio of the specific angular momentum $|j|$ of the gas and the largest eigenvalue $a_{1}$ of the inertia tensor, corresponding to the largest axis of the system ${ }^{60}$. The center of these profiles are taken to be the maximum gas density, consistent with the rest of our analysis. We compare this rotational velocity against the Keplerian velocity $v_{\mathrm{Kep}}=\sqrt{G M / r}$. The regions a

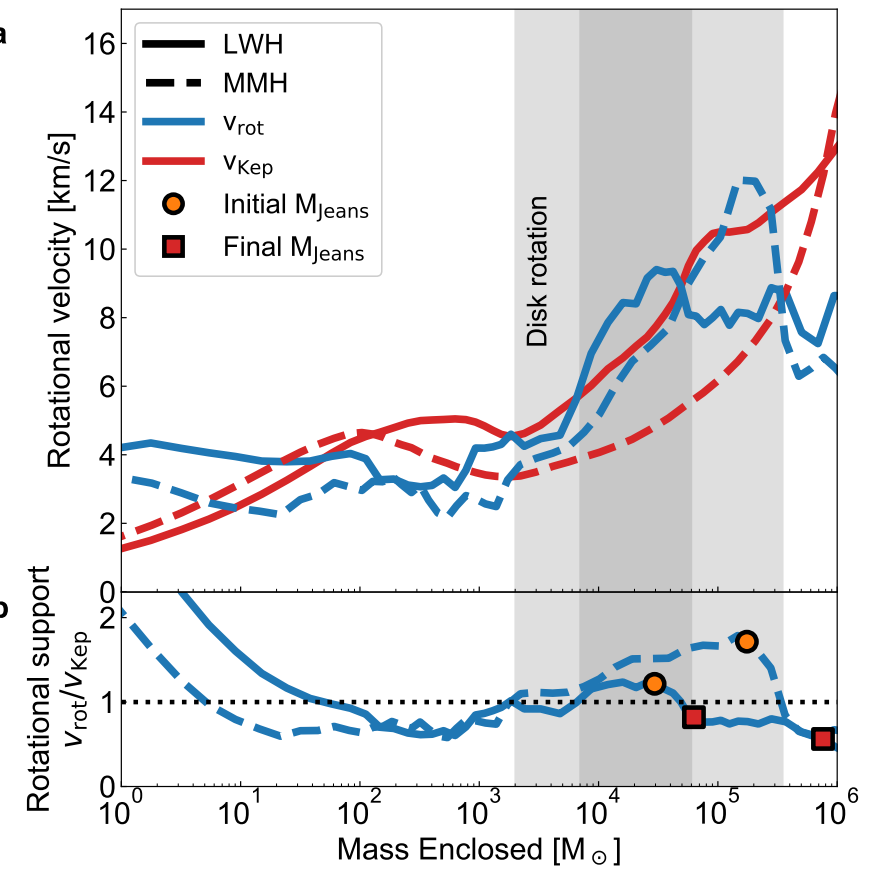

Extended Data Figure 4 Rotational properties of the target haloes. a, Radially averaged profiles of circular velocity $v_{\text {Kep }}=\sqrt{G M / r}$ (red lines) and rotational velocity $v_{\text {rot }}$ (blue lines) around the largest principal axis of the $\mathrm{MMH}$ (dashed lines) and LWH (solid lines) at the end of the simulation. b, Radially averaged profiles of the fractional rotational support, where a ratio greater than one indicates rotational velocities are sufficient to prevent gravitational collapse. The shaded regions show where the systems are rotationally supported, spanning from 2,000 to $330,000(7,000$ to 60,000$)$ solar masses for the MMH (LWH). Rotation works in tandem with thermal and turbulent pressures to marginally slow the collapse, seen in the lower infall speeds at these mass scales in Extended Data Fig. 3. Inside 100 solar masses, this rotational measure becomes ill-defined because the rotation center and center-of-mass are not co-located, thus we do not conclude that the inner portions are rotationally supported even though $v_{\text {rot }} / v_{\text {Kep }}>1$.

where the rotational velocity exceeds the Keplerian velocity are shaded and indicate regions of rotational support. The rotationally supported region in the MMH extends from 2,000 to $330,000 \mathrm{M}_{\odot}$. For the $\mathrm{LWH}$, the region of rotational support is significantly smaller spanning only from 7,000 to $60,000 \mathrm{M}_{\odot}$. The rotational velocities within $100 \mathrm{M}_{\odot}$ are not well defined because the separation between the densest gas parcel and rotation center becomes comparable to the radius $(0.02 \mathrm{pc})$ enclosing $100 \mathrm{M}_{\odot}$. We thus do not consider them to be rotationally supported from this analysis. The (disklike) regions of rotational support are nonetheless unstable to fragmentation and as we will see fragmentation of the disk does occur in the MMH.

Fragmentation of the collapsing core into gas clouds. As the cloud collapses, cooling instabilities can cause the gas to fragment into selfgravitating clumps. Such fragmentation can be stabilized by thermal pressure or centrifugal forces that can quantified by the Toomre parameter,

$$
Q \equiv \frac{c_{\mathrm{s}} \kappa}{\pi G \Sigma}
$$

The system is stable if $Q>1$. Here $\Sigma$ is the gas surface density, and the epicyclic frequency $\kappa$ is directly calculated from the data as

$$
\kappa^{2}=\frac{2 \Omega}{r} \frac{d\left(r^{2} \Omega\right)}{d r},
$$

where $\Omega=v_{\text {rot }} / r$ is the angular velocity, and $r$ is the radius in cylindrical coordinates with the $z$-axis aligned with the total angular momentum vector of the Jeans unstable gas cloud. These expressions assume an axisymmetric object. However, the collapsed objects in both haloes have non-ideal geometries. It is thus beneficial to consider a local measure of stability ${ }^{26}$

$$
Q_{\text {local }} \simeq \frac{\Omega^{2}}{\pi G \rho}
$$




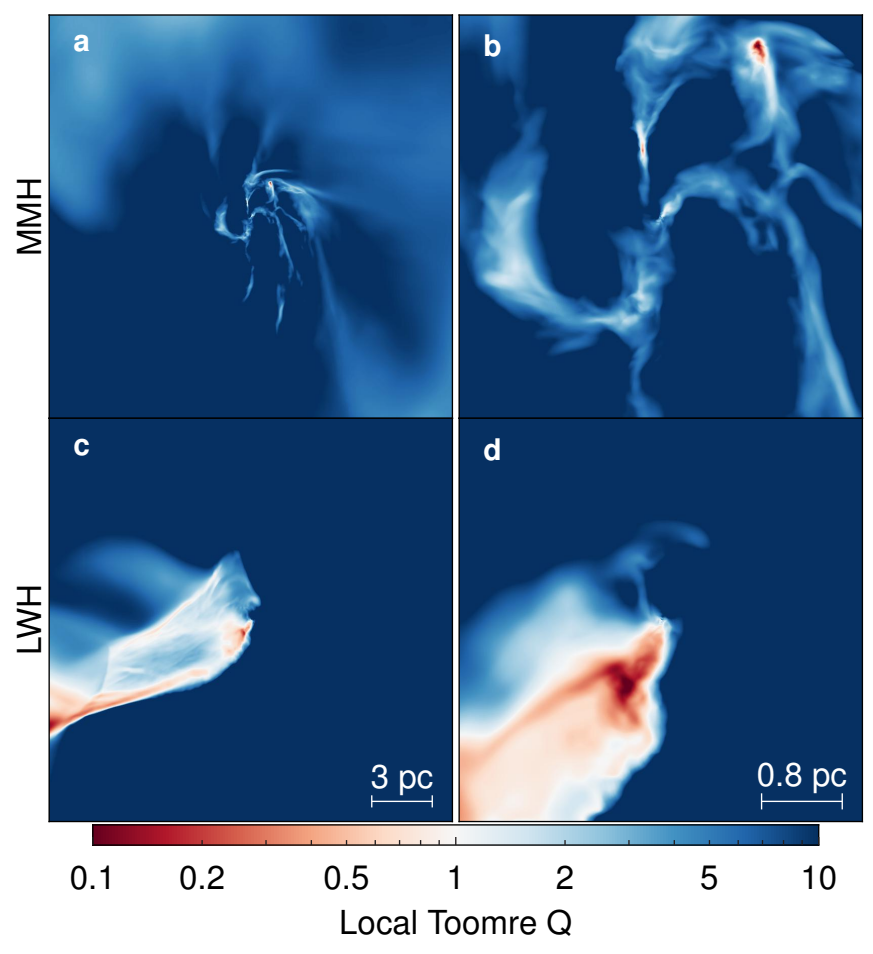

Extended Data Figure 5 | Distribution of fragmentation-prone regions. Density-weighted projections of a local estimate of the Toomre $Q$ parameter (Eq 7) for the MMH (a,b) and LWH (c,d) in a field of view of 20 (left) and 4 (right) parsecs, centered on the densest point and aligned to be perpendicular with the angular momentum vector of the disk. A value greater than one indicates that rotation and internal pressure stabilizes regions against fragmentation into smaller self-gravitating objects. In the MMH, this analysis highlights the clump fragments with the filaments being only marginally stable at $Q \sim 1$. The sheet in the LWH formed from a preceding major halo merger is apparent in this measure. The bulk of the sheet is only marginally stable with the edge and collapsing center containing an environment conducive toward fragmentation.

that can identify unstable regions with arbitrary geometries. Extended Data Fig. 5 shows $Q_{\text {local }}$ for both haloes in fields of view of 20 and 4 pc. In the MMH, the disk-like object is marginally stable in the spiral overdensities (panel a). Within those arms, there exists three clumps that have fragmented (panel b) and started to collapse. In the LWH, the overdense sheet-like object, which is induced by a major merger, is unstable to fragmentation in many regions and marginally stable in the remainder of the object. However, their collapse times are spread out, resulting in only one (the first) clump to fragment out of the overdense sheet by the end of the simulation.

Extended Data Fig. 6a shows the Toomre Q parameter as a function of cylindrical radius, where we have aligned the cylindrical $z$-axis with the total angular momentum vector of the inner $10 \mathrm{pc}$. We compare it with the effective Toomre $\mathrm{Q}$ parameter $Q_{\text {eff }}$, where we substitute $c_{\mathrm{S}}$ with $c_{\text {eff }}$ to include any turbulent pressure support in the stability analysis. When only considering rotational support as a counterbalance to gravitational collapse, the MMH and LWH are unstable $(Q<1)$ within a radius of three and eight pc, respectively. As we have shown earlier, turbulent pressures are comparable to thermal pressures, and this additional support stabilizes the rotating system against fragmentation $\left(Q_{\text {eff }}>1\right)$ outside 0.8 and $0.1 \mathrm{pc}$ in the MMH and $\mathrm{LWH}$. The $\mathrm{LWH}$ is also susceptible to fragmentation at radii of 0.8 and $2 \mathrm{pc}$, where $Q_{\text {eff }}$ becomes slightly less than unity.

From the dispersion relation

$$
w_{\mathrm{g}}^{2}=\kappa^{2}+k^{2} v_{\mathrm{rot}}^{2}-2 \pi G k \Sigma
$$

that is used to calculate the growth perturbations in a gaseous disk, the asso-

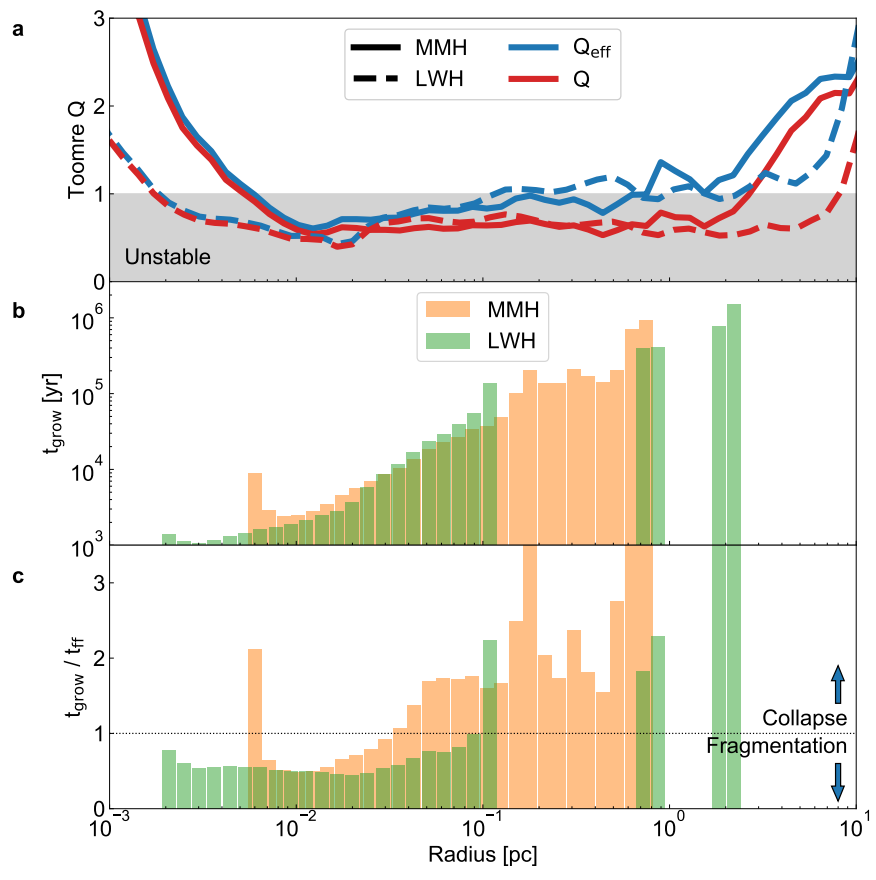

Extended Data Figure 6 | Growth rates for fragmentation. A rotating system will only fragment into self-gravitating clumps when their growth rates are faster than the collapse timescale. a, Cylindrical radial profiles of the Toomre Q parameter when considering only thermal support (red) and with thermal and turbulent support (blue) for the MMH (dashed) and LWH (solid). The shaded region indicates where the system is unstable to fragmentation. $\mathbf{b}$, The unstable regions have a characteristic growth rate, defining a growth timescale $t_{\text {grow }}$, showing an increasing trend with radius for the MMH (orange) and LWH (green). c, If the ratio of $t_{\text {grow }}$ and the free-fall time $t_{\mathrm{ff}}$ is less than one, the region can fragment before it gravitationally collapses. In the MMH, this condition is true at radii less than 0.03 parsecs, indicating that small-scale fragmentation might occur but will be suppressed subsequently by a rapid monolithic collapse. The LWH exhibits this feature inside 0.1 parsecs but is surrounded by gas that is stable against fragmentation.

ciated maximum growth rate ${ }^{61}$ of a perturbation is given by

$$
\left|w_{\max }\right|=\frac{\kappa\left(1+Q_{\text {eff }}^{2}\right)^{1 / 2}}{Q_{\text {eff }}},
$$

that occurs at $k_{\max }=\kappa / c_{\text {eff }} Q$. Here $k$ is the wavenumber, and $w_{\mathrm{g}}$ is the growth rate. We define the growth time $t_{\text {grow }} \equiv 1 / w_{\max }$ where $Q_{\text {eff }}<1$ (Extended Data Fig. 6b). For such a perturbation to form before the system collapses, the growth time must be smaller than a free-fall time (Extended Data Fig. 6c), i.e. $t_{\text {grow }} / t_{\mathrm{ff}}<1$. For the MMH, this condition is valid for radii smaller than $0.03 \mathrm{pc}$, corresponding to $100 \mathrm{M}_{\odot}$ of enclosed gas mass. Between this radius and $1 \mathrm{pc}$, the system is unstable to fragmentation, but it is collapsing faster than it can fragment. This analysis is centered on the densest point, contained in one of the clumps. This indicates that any fragmentation at small scales will be surrounded by a monolithic rapid collapse, most likely suppressing further fragmentation as this matter falls inward. The two other clumps in the MMH form about $1 \mathrm{pc}$ away. Because we compute these quantities within cylindrical shells, the clumps with small $Q_{\text {local }}$ (see Extended Data Fig. 5) are averaged out. Once they fragment, they begin to gravitationally collapse. In the LWH, fragmentation can occur before collapse inside a radius of $0.1 \mathrm{pc}$. Here a single clump fragments from the sheet-like overdensity, produced by a recent major merger, with a radius of $\sim 1 \mathrm{pc}$, which collapses faster than it can fragment at this length scale. This analysis shows that turbulence plays a role in providing additional stabilization against fragmentation. The suppression of fragmentation allows for only a few clumps, at most, to form in the collapsing system before the most dense clump collapses on the order of its free-fall time, $t_{\mathrm{ff}} \sim 5,000$ yr. This behavior allows for the rapid infall to be concentrated on these few 
clumps, strengthening the case of SMS formation.

Given that the system is unstable to fragmentation, we search for selfgravitating clouds with the clump finder provided with the analysis toolkit $\mathrm{yt}^{29}$. We search for clumps that are gravitationally bound and have a minimum of 20 cells, where clumps are defined as topologically connected sets of cells and form a hierarchical set of objects. Because the LWH only has one clump that contains the densest point, we only perform this analysis on the MMH. We identify three clumps that are also visible in the density projections (Fig. 3b). Below we have defined $t$ as the time before collapse, where $t=0$ is a free-fall time after the final simulation output of the maximum density. The three clumps are orbiting in the disk, located $1-2 \mathrm{pc}$ from the rotational center, where there is no local density maximum present.

- Clump 1 contains the densest point in the simulation, and the projections (Fig. 3; Extended Data Fig. 5) and global radial profiles (Fig. 4; Extended Data Fig. 2, 3, 4, 6) are centered on it. Its mass initially fluctuates between 200 and $600 \mathrm{M}_{\odot}$ at $t=30-70 \mathrm{kyr}$ as two clumps orbit each other, changing between topologically connected and disconnected in the process. Their separation decays as energy is dissipated during their close encounters, eventually merging into a single clump at $t=25 \mathrm{kyr}$. It grows to a mass of $790 \mathrm{M}_{\odot}$ and is located 1.2 $\mathrm{pc}$ from the rotational center at the final time.

- Clump 2 is an elongated object that steadily grows in mass from 200 to $840 \mathrm{M}_{\odot}$, orbiting at a distance of $1.8 \mathrm{pc}$, located above the Clump 1 in the projections.

- Clump 3 initially has a mass of $770 \mathrm{M}_{\odot}$, orbiting at a distance of 2.2 $\mathrm{pc}$, located in the upper-right of the projections. At $t=50 \mathrm{kyr}$, it merges with another clump, increasing its mass from 950 to $1400 \mathrm{M}_{\odot}$ The change in its distance from the rotation center is associated with the change in its center of mass after the merger. It grows very little afterwards to a final mass of $1500 \mathrm{M}_{\odot}$, while it migrates outward from 1.6 to $1.9 \mathrm{pc}$.

As the clumps evolve, they do not migrate inwards toward the disk center, suggesting that mergers are not imminent between these three clumps. This strengthens the argument for 3 separate sites of SMS formation and potentially DCBH formation. Such systems may be detectable in the future through gravitational waves ${ }^{62}$.

Collapse characteristics of the fragments. We now examine the accretion rates and thermal support of the clumps that form in both the MMH and LWH. In Extended Data Fig. 7a, we plot the infall rate of the gas versus the enclosed mass for each of the clumps (three for the MMH and one for the LWH). The infall rates are computed as the mass flux through spherical shells and show a steady and near monotonic increase in infall rate as a function of enclosed mass. The infall rate for the LWH is more than a factor of three greater than the three major clumps identified within the MMH. This is likely due to the increased mass, at collapse time, and the higher radial velocity associated with the LWH. The LWH experiences a major merger less than $10 \mathrm{Myr}$ before the end of the simulation that drives gas towards the center thus explaining the increased infall-rates.

Circles in panel (a) mark the infall rate at the clump mass. This is the infall rate that the outer part of the gas clump experiences. The rates onto each clump are very similar with values between 0.01 and $0.1 \mathrm{M}_{\odot} \mathrm{yr}^{-1}$. These values are consistent with the values obtained for a singular isothermal sphere collapse originally investigated by Shu $(1977)^{63}$. However, it should be noted that this is the infall rate onto the outer gas clump and must be treated as an upper limit when ascribing it to the accretion rate onto a protostar which may subsequently form within the gas clump. Nonetheless, the infall rates are very high and sufficient to drive a protostar towards SMS formation.

In panel (b) we plot the infall time against enclosed mass, which is calculated as the ratio of the mass enclosed and infall rate. It can be used to directly compare against star formation timescales. Within an enclosed mass of $100 \mathrm{M}_{\odot}$ the timescale for each clump is approximately $10 \mathrm{kyr}$. The Kelvin-Helmholtz timescale for massive stars $\left(\mathrm{M}_{\star} \lesssim 100 \mathrm{M}_{\odot}\right)$ is less than $100 \mathrm{kyr}$. The stars that form within these gas clumps will therefore reach the main sequence while still accreting as is the case in SMS formation. Given the timescales shown here, the infall rates suggest that the (super)massive

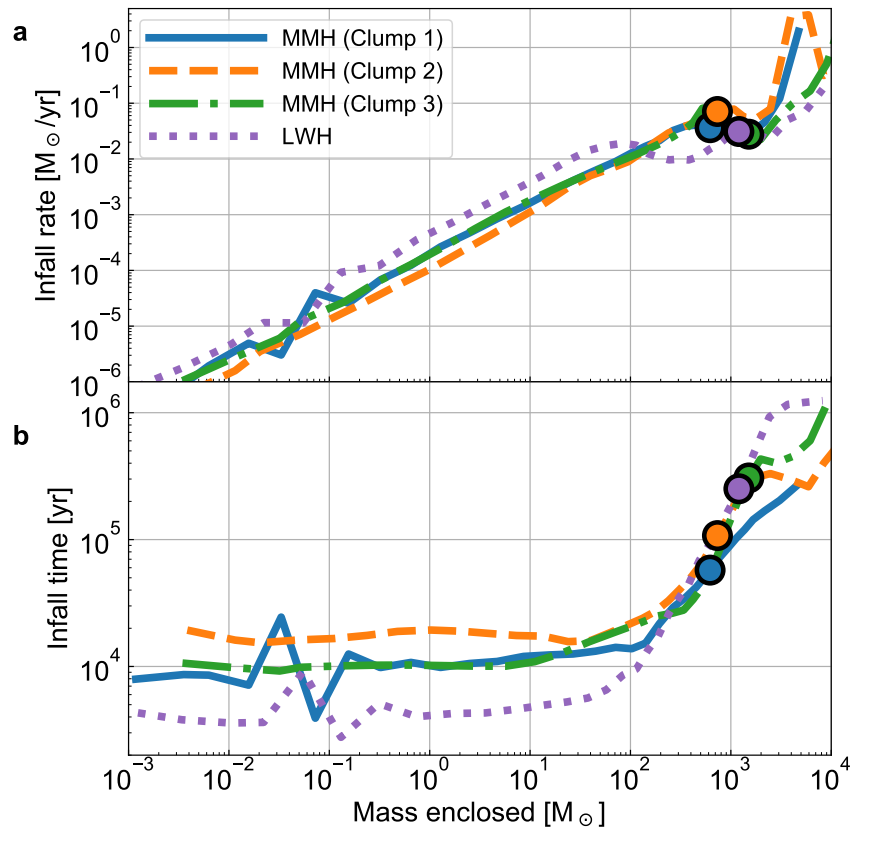

Extended Data Figure 7 | Clump infall rates and timescales. Similar to the results presented in Fig. 4d, the self-gravitating clumps are growing through radial infall. a, The infall rates are computed as the mass flux through spherical shells, showing a steadily increasing rate with enclosed mass. The rate in the single clump of the LWH (dotted purple line) is more than a factor three greater than the three major clumps in the $\mathrm{MMH}$. The circles mark the infall rate at the clump mass. b, The infall time, the ratio of the mass enclosed and infall rate, is an informative scale that can be used to compare against star formation timescales. This timescale is constant and approximately 10,000 years within 100 solar masses for all clumps and rises to $\sim 100,000$ years for the entire clump, marked by the circles. This rapid infall suggests that sufficient mass will collapse into supermassive protostar before it reaches main sequence.

protostars will reach masses of at least $100 \mathrm{M}_{\odot}$ before reaching the main sequence ${ }^{64}$. If the accretion onto the clump continues to remain rapid at that point, full gravitational contraction will be avoided (so-called "hot accretion" flows), and the stellar envelope will remain bloated, leading to SMS formation in this context ${ }^{5,9,65,66}$. After its formation, SMS lifetimes are around $1 \mathrm{Myr}$, suggesting that the whole gas cloud could be accreted before the star exhausts its hydrogen supply. SMSs with accretion rates below 0.1 solar masses per year experience a general relativistic instability, creating a massive black hole with a mass similar to its progenitor mass, when its nuclear fuel is exhausted. For higher accretion rates, the collapse occurs when the star is still burning hydrogen or helium ${ }^{10}$, producing black holes with masses between 200,000 and $800,000 \mathrm{M}_{\odot}$. Lastly, there is a peculiar case when the SMS has a mass of around $55,000 \mathrm{M}_{\odot}$ that produces an extremely energetic supernova ${ }^{67}$.

In Extended Data Fig. 8, we plot the fractional support against collapse, similar to Extended Data Fig. 3. We do this by again comparing the thermal, turbulent and infall velocities of the clumps. Panel (a) shows the velocities of the clump found for the LWH. Panels (b), (c), and (d) show the clump velocities of each of the three clumps found for the MMH. We find that in all cases the clumps are thermally supported and stable against further gravitational collapse. The radial inflows are subsonic for all four clumps, however the clump in the LWH contains transonic flows between $100 \mathrm{M}_{\odot}$ and 1000 $\mathrm{M}_{\odot}$. We also note that the thermal pressure support is strongly dominant over turbulent pressure support in all four clumps. This is in contrast to the Jeans-unstable parent cloud where the turbulent pressures were dominant.

Estimating the number density of SMS/DCBH formation sites. We estimate the comoving number density of $\mathrm{DCBH}$ formation sites, $n_{\mathrm{DCBH}}$, through the following expression: $n_{\mathrm{DCBH}}=n_{\mathrm{ACH}} f_{\mathrm{prim}} f_{\mathrm{LW}} f_{\text {rapid }}$. Here $n_{\mathrm{ACH}}=5.0 \pm 0.19$ per comoving $\mathrm{Mpc}^{3}$ is the comoving number 


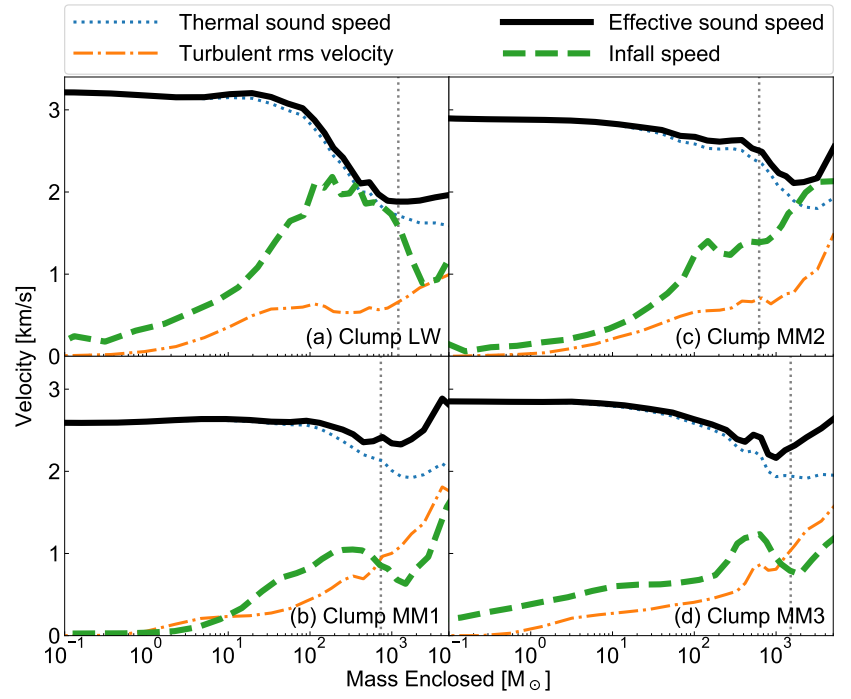

Extended Data Figure 8 Thermal and turbulent support of collapsing clumps. Same as Extended Data Figure 3 but for the clumps in the LWH (a) and MMH (b-d). The vertical dotted lines mark the clump mass in each panel. The radial inflows are subsonic for all four clumps, however the clump in LWH contains transonic flows between 100 and 1,000 solar masses. Thermal support is dominant inside the clumps, unlike the larger parent Jeans-unstable system where turbulent effective pressures are comparable to their thermal counterparts (see Extended Data Figure 3).

density of atomic-cooling haloes at redshift $z=15$ (i.e., haloes above the mass threshold where cooling via atomic hydrogen is effective); $f_{\text {prim }}=$ $0.015 \pm 0.0045$ is the fraction of those haloes that are of primordial composition; $f_{\mathrm{LW}}=0.1 \pm 0.05$ is the fraction of the atomic cooling haloes that are in regions where $\mathrm{J}_{21} \geq 3$ (i.e., the fraction of the simulation volume above that flux threshold); and $f_{\text {rapid }}=0.20 \pm 0.14$ is the fraction of the primordial atomic cooling haloes that are growing above the critical threshold where accretion heating is greater than cooling. Based on the quantities found in the rare-peak region of our simulation, we estimated these values and their variance, which are given in the main article. We then perform a Monte Carlo sampling of the parameter space. Extended Data Fig. 9 shows the cumulative probability of the expected DCBH number density in different large-scale environments. They steeply decrease above $10^{-3}, 10^{-6}$, and $10^{-9}$ DCBHs per comoving cubic megaparsec in the rare-peak, normal, and void regions of the Renaissance simulations. The rare-peak has a median value of $n_{\mathrm{DCBH}}=1.1 \times 10^{-3}$ haloes $/ \mathrm{cMpc}^{3}$, a $68 \%$ confidence interval of $1.9 \times 10^{-4}-2.8 \times 10^{-3}$ haloes $/ \mathrm{cMpc}^{3}$, and a $95 \%$ confidence interval of $0-5.2 \times 10^{-3}$ haloes $/ \mathrm{cMpc}^{3}$ in the rare-peak region, where "cMpc" denotes comoving Mpc.

We note that the values described above represent the rare-peak region only. When the same quantities are extracted from the normal and void simulation regions at $z=15$, the median number density of DCBH haloes is expected to be $\sim 10^{-7}$ haloes $/ \mathrm{cMpc}^{3}$ and 0 haloes/cMpc ${ }^{3}$, respectively, due to both the lack of atomic cooling haloes of primordial composition and a lack of atomic cooling haloes that rapidly grow. The rare-peak region has a volume of $133.6 \mathrm{cMpc}^{3}$ (equivalent to a sphere of approximately 2.25 $h^{-1} \mathrm{cMpc}$ in radius) and an average density of 1.65 times the cosmic mean. At $z=15$, regions of that size and overdensity are expected to be found in approximately $10^{-4}-10^{-3}$ of the volume of the universe, implying that a more realistic estimate of the global number density of DCBH candidates forming through this mechanism is in the range of $10^{-7}-10^{-6}$ haloes $/ \mathrm{cMpc}^{3}$, consistent with our estimate in the normal region. This estimate is nonetheless approximately $100-1000$ times greater than the observed $z \sim 6$ quasar number density ${ }^{27}$. Our results suggest that faint quasars at these high redshifts should be strongly clustered and associated with galaxy overdensities ${ }^{68}$, providing an observational test of our proposed seeding mechanism. Furthermore, we find that DCBHs forming in this sce-

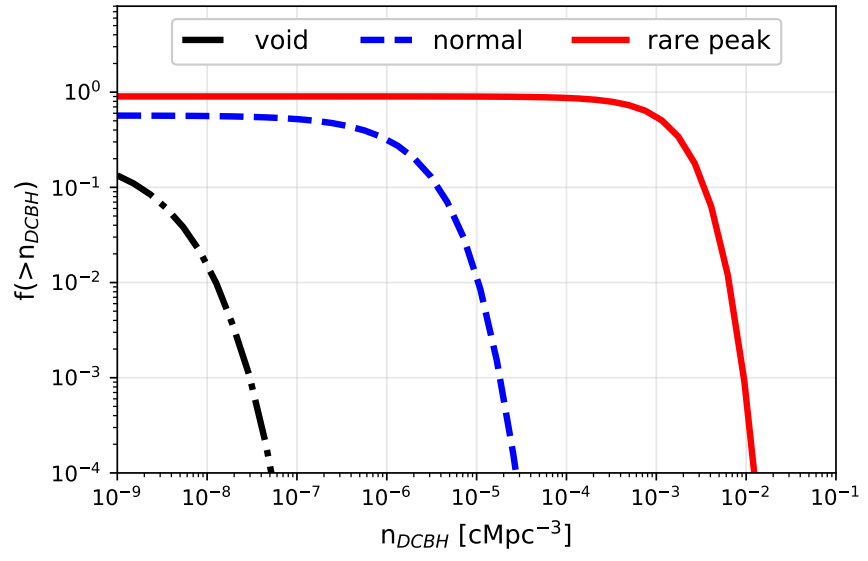

Extended Data Figure 9 | Abundance estimate of direct collapse black holes. The cumulative probability of the halo comoving number density that potentially host supermassive star formation shown for the rare-peak (red solid line), normal (blue dashed), and void (black dash-dotted) regions of the Renaissance Simulations. Their respective median number densities are $1.1 \times 10^{-3}, \sim 10^{-7}$, and 0 per comoving cubic megaparsec. Subsequent direct collapse black hole formation are most likely to occur in overdense regions of the early universe, whereas little to no will form in average and underdense regions.

nario have the same number density as the observed number density ${ }^{69,70}$ of present-day SMBHs above $10^{8} \mathrm{M}_{\odot}$. This abundance matching intriguingly suggests that such central black holes in most elliptical galaxies have a common origin beginning their lives as SMSs.

The fraction of rapidly growing atomic cooling haloes. We compute an additional check on the fraction of rapidly growing haloes with semianalytical galaxy formation code GALACTICUS ${ }^{71}$. Most of the rare-peak region collapses into a halo with a total mass $8.5 \times 10^{11} \mathrm{M}_{\odot}$ at $z=5$. We calculate 50 merger trees of equivalent $z=5$ haloes that follow progenitor haloes as small as $3 \times 10^{5} \mathrm{M}_{\odot}$. Each merger tree has approximately 40,000 progenitor haloes at $z=15$, totaling 2 million progenitors in all of the calculated merger trees at this epoch. We find that $3 \times 10^{-4}$ of haloes around the atomic cooling threshold, $M_{\text {halo }}=(3-6) \times 10^{7} \mathrm{M}_{\odot}$, grow more than a factor of six between redshifts 16 and 15 ( $\sim 20 \mathrm{Myr})$. By using the halo descendant at a much later time, we can incorporate the effects of being in an overdense environment that leads to higher halo mass growth rates. This leads to a more accurate estimate when compared to randomly sampling atomic cooling haloes at $z=15$ whose merger trees would be unlikely to be representative of our target haloes.

The metal-free haloes found in the Renaissance Simulations. We searched and have reported on the final output at $z=15$ of the rare-peak region in the Renaissance simulation that satisfied the following criteria: (1) an atomic cooling halo $\left(M_{\text {halo }} \geq 4.9 \times 10^{7} \mathrm{M}_{\odot}\right.$ at $\left.z=15\right)$, (2) only contains high-resolution dark matter particles, (3) does not support metal or dust radiative cooling $\left(Z<10^{-6} \mathrm{Z}_{\odot}\right)$, and (4) no prior star formation. We consider the last criterion because some Pop III stars directly collapse into a stellar-mass black hole, producing no metals ${ }^{72,73}$; however because we randomly sample from an initial mass function, it is just as likely that the same Pop III star particle in question could have chemically enriched its host halo. Out of 670 atomic cooling haloes, ten haloes fit these conditions with their details shown in Extended Data Table 1. We also searched the "normal" and "void" regions and found no haloes matching the same criteria at $z=15$. This suggests that SMS formation through this channel is much more likely in overdense regions of the early universe. This crowded high-redshift environment lends itself to high LW radiation field from more nearby galaxies and haloes with very high growth rates, existing in a medium with a higher average mass density. This null detection agrees with our statistical expectations discussed earlier.

We have focused on the most massive and most irradiated haloes in the main article, but from the other eight haloes, we can gain insight on their variations. Eight of ten haloes experienced growth rate greater than 
Table 1 Properties of halo candidates hosting supermassive star formation

\begin{tabular}{cccccc}
\hline $\begin{array}{c}\log _{10}\left(\mathrm{M}_{\text {halo }}\right) \\
{\left[\mathrm{M}_{\odot}\right]}\end{array}$ & $\begin{array}{c}\log _{10}(\text { mean Growth rate }) \\
{\left[\mathrm{M}_{\odot} \text { per unit redshift }\right]}\end{array}$ & $\mathrm{J}_{\mathrm{LW}} / \mathrm{J}_{21}$ & $\begin{array}{c}\mathrm{D}_{\text {gal }} \\
{[\mathrm{kpc}]}\end{array}$ & $\begin{array}{c}\mathrm{T}_{\mathrm{c}} \\
{[\mathrm{K}]}\end{array}$ & $\begin{array}{c}\text { Gas infall rate } \\
{\left[\mathrm{M}_{\odot} / \mathrm{yr}\right]}\end{array}$ \\
\hline $7.84^{*}$ & 7.78 & 2.71 & 12.7 & 2250 & 0.275 \\
$7.76^{\dagger}$ & 7.53 & 3.23 & 11.8 & 4390 & 0.171 \\
7.76 & 7.76 & 1.91 & 14.7 & 4220 & 0.286 \\
7.75 & 7.65 & 0.583 & 35.5 & 1730 & 0.290 \\
7.75 & 7.39 & 0.958 & 19.7 & 7570 & 0.0294 \\
7.74 & 7.88 & 1.49 & 25.0 & 8670 & 0.0574 \\
7.73 & 7.79 & 0.894 & 29.9 & 1760 & 0.396 \\
7.70 & 7.22 & 2.62 & 18.3 & 6520 & 1080 \\
7.67 & 7.90 & 0.16 & 6.20 & 7890 & 0.0292 \\
7.64 & 7.78 & 2.14 & & 0.0356 \\
\hline
\end{tabular}

Table notes: ${ }^{*}$ Most massive halo (MMH). ${ }^{\dagger}$ Halo exposed to the highest Lyman-Werner radiation flux (LWH). The growth rate is averaged over the last 20 million years of the simulation. The values of $\mathrm{J}_{\mathrm{LW}}$, Lyman-Werner intensity in units of $\mathrm{J}_{21}$, and $\mathrm{T}_{\mathrm{c}}$, the gas temperature, are given at the densest point. $\mathrm{D}_{\text {gal }}$ is the distance to the nearest galaxy with at least $10^{6} \mathrm{M}_{\odot}$ of stars. The gas infall rate is the mass-averaged value within 100 parsecs. All data are given at redshift 15 from the original Renaissance Simulation of the rare-peak region.

$10^{7.5} \mathrm{M}_{\odot}$ per unit redshift, showing that they grow from the minimum mass $M_{\text {crit }}$ for molecular hydrogen cooling to one supporting atomic cooling in less than a dynamical time ( $20 \mathrm{Myr})$. With the exception of one halo, they have a galaxy with a stellar mass over $10^{6} \mathrm{M} \odot$ within 40 proper kpc. Stars in nearby galaxies create a boosted LW radiation field above $0.5 J_{21}$. Inspecting the central temperatures, we see that there are six cool core $(T<5000 \mathrm{~K})$ haloes that have efficient $\mathrm{H}_{2}$ cooling, whereas the other four haloes still have warm cores. For various reasons, such as the LW radiation field and the degree of turbulence in the core ${ }^{46}$, the warm core haloes have their cooling suppressed at the simulation's final redshift. However, this state is temporary, and the system will cool within a timescale defined by the ratio of the $\mathrm{H}_{2}$ fraction and its formation rate as the haloes continue to grow ${ }^{46}$. The higher core pressure limits the gravitational collapse, resulting in lower infall rates $\left(<0.1 \mathrm{M}_{\odot} \mathrm{yr}^{-1}\right)$ when compared to the cool core haloes. The infall rates in our SMS candidate list do not follow the usual thermal accretion rate $\left(c_{\mathrm{S}}^{3} / G\right)$ because they systems have lost their thermal support in a short timescale relative to the free-fall time. Thus, they are experiencing their initial catastrophic collapse. Although SMS models have shown that a critical infall rate of $0.04 \mathrm{M}_{\odot} \mathrm{yr}^{-1}$ is required, we include these warm core haloes in our candidate list because molecular cooling and thus a further and more rapid collapse is inevitable ${ }^{74}$. The halo with the highest infall rate $\left(\sim 1 \mathrm{M}_{\odot} \mathrm{yr}^{-1}\right)$ is an interesting case because it exists the farthest (124 proper kpc) from a galaxy and thus has the lowest $J_{\mathrm{LW}}$ value in this sample. This halo also experiences the most rapid growth rate, which we attribute to it not collapsing earlier before it reaches the atomic cooling limit. We also note here that the accretion rates given are for a single snapshot, and the average accretion rate will vary as such haloes undergo quiescent and intensive periods of cosmological accretion.

Data Availability. The numerical experiments presented in this work were run with a hybrid OpenMP+MPI fork of the ENZO code available from https://bitbucket.org/jwise77/openmp, using the changeset bcb436949d16. The data are publicly available from the Renaissance Simulation Laboratory at http: //girder.rensimlab.xyz.

30. Abel, T., Bryan, G. L. \& Norman, M. L. The Formation of the First Star in the Universe. Science 295, 93-98 (2002). arXiv: astro-ph/ 0112088.

31. O'Shea, B. W. \& Norman, M. L. Population III Star Formation in a $\Lambda$ CDM Universe. I. The Effect of Formation Redshift and Environment on Protostellar Accretion Rate. Astrophys. J. 654, 66-92 (2007). arXiv: astro-ph/0607013.

32. Turk, M. J., Abel, T. \& O'Shea, B. The Formation of Population III Binaries from Cosmological Initial Conditions. Science 325, 601- (2009). 0907. 2919.

33. Xu, H., Wise, J. H. \& Norman, M. L. Population III Stars and Remnants in High-redshift Galaxies. Astrophys. J. 773, 83 (2013). 1305.1325.
34. Xu, H., Ahn, K., Wise, J. H., Norman, M. L. \& O'Shea, B. W. Heating the Intergalactic Medium by X-Rays from Population III Binaries in Highredshift Galaxies. Astrophys. J. 791, 110 (2014). 1404.6555.

35. Chen, P., Wise, J. H., Norman, M. L., Xu, H. \& O'Shea, B. W. Scaling Relations for Galaxies Prior to Reionization. Astrophys. J. 795, 144 (2014). 1408.2523.

36. Ahn, K., Xu, H., Norman, M. L., Alvarez, M. A. \& Wise, J. H. Spatially Extended $21 \mathrm{~cm}$ Signal from Strongly Clustered Uv and X-Ray Sources in the Early Universe. Astrophys. J. 802, 8 (2015). 1405.2085.

37. Xu, H., Norman, M. L., O'Shea, B. W. \& Wise, J. H. Late Pop III Star Formation During the Epoch of Reionization: Results from the Renaissance Simulations. Astrophys. J. 823, 140 (2016). 1604.03586.

38. Komatsu, E. et al. Seven-year Wilkinson Microwave Anisotropy Probe (WMAP) Observations: Cosmological Interpretation. Astrophys. J. Suppl. 192, 18 (2011). 1001.4538.

39. Hahn, O. \& Abel, T. Multi-scale initial conditions for cosmological simulations. Mon. Not. R. Astron. Soc. 415, 2101-2121 (2011). 1103.6031.

40. Abel, T., Anninos, P., Zhang, Y. \& Norman, M. L. Modeling primordial gas in numerical cosmology. New Astronomy 2, 181-207 (1997).

41. Smith, B. D., Turk, M. J., Sigurdsson, S., O'Shea, B. W. \& Norman, M. L. Three Modes of Metal-Enriched Star Formation in the Early Universe. Astrophys. J. 691, 441-451 (2009). 0806.1653.

42. Behroozi, P. S., Wechsler, R. H. \& Wu, H.-Y. The ROCKSTAR Phasespace Temporal Halo Finder and the Velocity Offsets of Cluster Cores. Astrophys. J. 762, 109 (2013). 1110.4372.

43. Behroozi, P. S. et al. Gravitationally Consistent Halo Catalogs and Merger Trees for Precision Cosmology. Astrophys. J. 763, 18 (2013). 1110. 4370.

44. Wise, J. H., Turk, M. J., Norman, M. L. \& Abel, T. The Birth of a Galaxy: Primordial Metal Enrichment and Stellar Populations. Astrophys. J. 745, 50 (2012). 1011.2632

45. Regan, J. A., Johansson, P. H. \& Wise, J. H. Forming Super-Massive Black Hole Seeds under the Influence of a Nearby Anisotropic MultiFrequency Source. Mon. Not. R. Astron. Soc. (2016). 1511.00696.

46. Wise, J. H. \& Abel, T. Suppression of $\mathrm{H}_{2}$ Cooling in the Ultraviolet Background. Astrophys. J. 671, 1559-1567 (2007). arXiv:0707.2059.

47. O'Shea, B. W. \& Norman, M. L. Population III Star Formation in a $\Lambda$ CDM Universe. II. Effects of a Photodissociating Background. Astrophys. J. 673, 14-33 (2008). 0706.4416.

48. Naoz, S., Yoshida, N. \& Gnedin, N. Y. Simulations of Early Baryonic Structure Formation with Stream Velocity. II. The Gas Fraction. Astrophys. J. 763, 27 (2013). 1207. 5515.

49. Regan, J. A., Johansson, P. H. \& Wise, J. H. The effect of dark matter resolution on the collapse of baryons in high-redshift numerical simulations. Mon. Not. R. Astron. Soc. 449, 3766-3779 (2015). 1501.05650.

50. Kitsionas, S. \& Whitworth, A. P. Smoothed Particle Hydrodynamics with particle splitting, applied to self-gravitating collapse. Mon. Not. R. Astron. Soc. 330, 129-136 (2002). astro-ph/ 0203057.

51. Bromm, V. \& Loeb, A. Formation of the First Supermassive Black Holes. Astrophys. J. 596, 34-46 (2003). arXiv: astro-ph/ 0212400.

52. Dotti, M., Colpi, M., Haardt, F. \& Mayer, L. Supermassive black hole binaries in gaseous and stellar circumnuclear discs: orbital dynamics and gas accretion. Mon. Not. R. Astron. Soc. 379, 956-962 (2007). astro-ph/0612505. 
53. Hirano, S. et al. One Hundred First Stars: Protostellar Evolution and the Final Masses. Astrophys. J. 781, 60 (2014). 1308.4456.

54. Chiaki, G. \& Yoshida, N. Particle splitting in smoothed particle hydrodynamics based on Voronoi diagram. Mon. Not. R. Astron. Soc. 451, 3955-3963 (2015).

55. Wolcott-Green, J., Haiman, Z. \& Bryan, G. L. Photodissociation of $\mathrm{H}_{2}$ in protogalaxies: modelling self-shielding in three-dimensional simulations. Mon. Not. R. Astron. Soc. 418, 838-852 (2011). 1106.3523.

56. Smith, B. D. et al. GRACKLE: a chemistry and cooling library for astrophysics. Mon. Not. R. Astron. Soc. 466, 2217-2234 (2017). 1610. 09591.

57. Barkana, R. \& Loeb, A. In the beginning: the first sources of light and the reionization of the universe. Phys. Rep. 349, 125-238 (2001). arXiv: astro-ph/0010468.

58. Wise, J. H. \& Abel, T. Resolving the Formation of Protogalaxies. I. Virialization. Astrophys. J. 665, 899-910 (2007). 0704.3629.

59. Wechsler, R. H., Bullock, J. S., Primack, J. R., Kravtsov, A. V. \& Dekel, A. Concentrations of Dark Halos from Their Assembly Histories. Astrophys. J. 568, 52-70 (2002). astro-ph/ 0108151.

60. Regan, J. A. \& Haehnelt, M. G. The formation of compact massive selfgravitating discs in metal-free haloes with virial temperatures of ${ }^{\sim 13000}$ 30000K. Mon. Not. R. Astron. Soc. 393, 858-871 (2009). 0810.0024.

61. Wang, B. \& Silk, J. Gravitational instability and disk star formation. Astrophys. J. 427, 759-769 (1994).

62. Hartwig, T., Agarwal, B. \& Regan, J. A. Gravitational wave signals from the first massive black hole seeds. Mon. Not. R. Astron. Soc. 479, L23L27 (2018).

63. Shu, F. H. Self-similar collapse of isothermal spheres and star formation. Astrophys. J. 214, 488-497 (1977).

64. Bonnell, I. A., Bate, M. R. \& Zinnecker, H. On the formation of massive stars. Mon. Not. R. Astron. Soc. 298, 93-102 (1998). astro-ph/ 9802332.

65. Hosokawa, T., Yorke, H. W. \& Omukai, K. Evolution of Massive Protostars Via Disk Accretion. Astrophys. J. 721, 478-492 (2010). 1005.2827.

66. Woods, T. E., Heger, A., Whalen, D. J., Haemmerlé, L. \& Klessen, R. S. On the Maximum Mass of Accreting Primordial Supermassive Stars. Astrophys. J. Lett. 842, L6 (2017). 1703.07480.

67. Chen, K.-J., Heger, A., Woosley, S., Almgren, A. \& Whalen, D. J. Pair Instability Supernovae of Very Massive Population III Stars. Astrophys. J. 792, 44 (2014). 1402.5960.

68. Ota, K. et al. Large-scale Environment of a $z=6.61$ Luminous Quasar Probed by Ly $\alpha$ Emitters and Lyman Break Galaxies. Astrophys. J. 856, 109 (2018). 1802.08912.

69. Shankar, F. The demography of supermassive black holes: Growing monsters at the heart of galaxies. New Astronomy Reviews 53, 57-77 (2009). 0907.5213.

70. Terrazas, B. A. et al. Quiescence Correlates Strongly with Directly Measured Black Hole Mass in Central Galaxies. Astrophys. J. Lett. 830, L12 (2016). 1609.07141.

71. Benson, A. J. GALACTICUS: A semi-analytic model of galaxy formation. New Astron. 17, 175-197 (2012). 1008.1786.

72. Heger, A., Fryer, C. L., Woosley, S. E., Langer, N. \& Hartmann, D. H. How Massive Single Stars End Their Life. Astrophys. J. 591, 288-300 (2003). arXiv: astro-ph/0212469.

73. Chatzopoulos, E. \& Wheeler, J. C. Effects of Rotation on the Minimum Mass of Primordial Progenitors of Pair-instability Supernovae. Astrophys. J. 748, 42 (2012). 1201.1328.

74. Visbal, E., Haiman, Z. \& Bryan, G. L. A no-go theorem for direct collapse black holes without a strong ultraviolet background. Mon. Not. R. Astron Soc. 442, L100-L104 (2014). 1403.1293. 